\title{
Updated global $3+1$ analysis of short-baseline neutrino oscillations
}

\author{
S. Gariazzo, ${ }^{a}$ C. Giunti, ${ }^{b}$ M. Laveder ${ }^{c, d}$ and Y.F. $\mathbf{L i}^{e, f}$ \\ ${ }^{a}$ Instituto de Física Corpuscular (CSIC-Universitat de València), \\ Paterna, Valencia, Spain \\ ${ }^{b}$ INFN - Sezione di Torino, \\ Via P. Giuria 1, 10125 Torino, Italy \\ ${ }^{c}$ Dipartimento di Fisica e Astronomia "G. Galilei", Università di Padova, \\ Padova, Italy \\ ${ }^{d}$ INFN - Sezione di Padova, \\ Via F. Marzolo 8, 35131 Padova, Italy \\ ${ }^{e}$ Institute of High Energy Physics, Chinese Academy of Sciences, \\ Beijing 100049, China \\ ${ }^{f}$ School of Physical Sciences, University of Chinese Academy of Sciences, \\ Beijing 100049, China \\ E-mail: gariazzo@ific.uv.es, giunti@to.infn.it, laveder@pd.infn.it, \\ liyufeng@ihep.ac.cn
}

ABSTRACT: We present the results of an updated fit of short-baseline neutrino oscillation data in the framework of $3+1$ active-sterile neutrino mixing. We first consider $\nu_{e}$ and $\bar{\nu}_{e}$ disappearance in the light of the Gallium and reactor anomalies. We discuss the implications of the recent measurement of the reactor $\bar{\nu}_{e}$ spectrum in the NEOS experiment, which shifts the allowed regions of the parameter space towards smaller values of $\left|U_{e 4}\right|^{2}$. The $\beta$-decay constraints of the Mainz and Troitsk experiments allow us to limit the oscillation length between about $2 \mathrm{~cm}$ and $7 \mathrm{~m}$ at $3 \sigma$ for neutrinos with an energy of $1 \mathrm{MeV}$. The corresponding oscillations can be discovered in a model-independent way in ongoing reactor and source experiments by measuring $\nu_{e}$ and $\bar{\nu}_{e}$ disappearance as a function of distance. We then consider the global fit of the data on short-baseline $\stackrel{(-)}{\nu_{\mu}} \rightarrow \stackrel{(-)}{\nu_{e}}$ transitions in the light of the LSND anomaly, taking into account the constraints from $\stackrel{(-)}{\nu_{e}}$ and $\stackrel{(-)}{\nu}$ disappearance experiments, including the recent data of the MINOS and IceCube experiments. The combination of the NEOS constraints on $\left|U_{e 4}\right|^{2}$ and the MINOS and IceCube constraints on $\left|U_{\mu 4}\right|^{2}$ lead to an unacceptable appearance-disappearance tension which becomes tolerable only in a pragmatic fit which neglects the MiniBooNE low-energy anomaly. The minimization of the global $\chi^{2}$ in the space of the four mixing parameters 
$\Delta m_{41}^{2},\left|U_{e 4}\right|^{2},\left|U_{\mu 4}\right|^{2}$, and $\left|U_{\tau 4}\right|^{2}$ leads to three allowed regions with narrow $\Delta m_{41}^{2}$ widths at $\Delta m_{41}^{2} \approx 1.7$ (best-fit), 1.3 (at $2 \sigma$ ), 2.4 (at $\left.3 \sigma\right) \mathrm{eV}^{2}$. The effective amplitude of short-baseline $\stackrel{(-)}{\nu} \rightarrow \stackrel{(-)}{\nu}$ oscillations is limited by $0.00048 \lesssim \sin ^{2} 2 \vartheta_{e \mu} \lesssim 0.0020$ at $3 \sigma$. The restrictions of the allowed regions of the mixing parameters with respect to our previous global fits are mainly due to the NEOS constraints. We present a comparison of the allowed regions of the mixing parameters with the sensitivities of ongoing experiments, which show that it is likely that these experiments will determine in a definitive way if the reactor, Gallium and LSND anomalies are due to active-sterile neutrino oscillations or not.

Keywords: Neutrino Physics, Beyond Standard Model

ARXIV EPRINT: 1703.00860 


\section{Contents}

1 Introduction 1

$2 \nu_{e}$ and $\bar{\nu}_{e}$ disappearance $\quad 3$

2.1 Reactor rates 3

2.2 Reactor spectra 9

2.3 Global $\nu_{e}$ and $\bar{\nu}_{e}$ disappearance $\quad 10$

$3 \quad$ Fits of appearance and disappearance data $\quad 12$

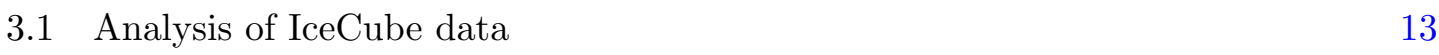

$\begin{array}{lll}3.2 & \text { Fit of the } 2016 \text { data set without MINOS and IceCube } & 17\end{array}$

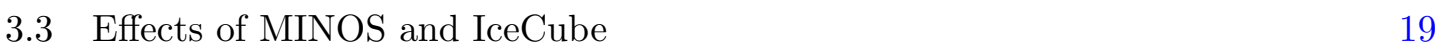

3.4 Effects of NEOS 21

3.5 Pragmatic fit 22

4 Conclusions $\quad 24$

\section{Introduction}

Neutrino physics is a powerful probe of new physics beyond the Standard Model. The LSND [1, 2], Gallium [3-7] and reactor [8] anomalies are intriguing indications in favor of the existence of light sterile neutrinos connected with low-energy new physics. In order to assess the viability of the light sterile neutrino hypothesis, it is necessary to perform a global fit of neutrino oscillation data which takes into account not only the LSND, Gallium and reactor anomalies, but also the data of many other experiments which constrain activesterile neutrino mixing (see the reviews in refs. [9-12]).

In this paper we consider $3+1$ active-sterile neutrino mixing, in which the three standard active neutrinos $\nu_{e}, \nu_{\mu}, \nu_{\tau}$ are mainly composed of three sub-eV massive neutrinos $\nu_{1}, \nu_{2}, \nu_{3}$ and there is a sterile neutrino $\nu_{s}$ which is mainly composed of a fourth massive neutrino $\nu_{4}$ at the $\mathrm{eV}$ scale. This is the only allowed four-neutrino mixing scheme after the demise of the $2+2$ scheme [13] and the fact that the $1+3$ scheme with three massive neutrinos at the $\mathrm{eV}$ scale is strongly disfavored by cosmological measurements [14] and by the experimental bounds on neutrinoless double- $\beta$ decay if massive neutrinos are Majorana particles (see refs. $[15,16]$ ). We do not consider neutrino mixing schemes with more than one sterile neutrino, which are not necessary to explain the current data (see the discussions in refs. $[12,17])$.

In the framework of $3+1$ active-sterile mixing, short-baseline (SBL) experiments are sensitive only to the oscillations generated by the squared-mass difference $\Delta m_{41}^{2} \simeq \Delta m_{42}^{2} \simeq$ $\Delta m_{43}^{2} \gtrsim 1 \mathrm{eV}^{2}$, with $\Delta m_{j k}^{2} \equiv m_{j}^{2}-m_{k}^{2}$, that is much larger than the solar squared-mass 
difference $\Delta m_{\text {SOL }}^{2}=\Delta m_{21}^{2} \approx 7.4 \times 10^{-5} \mathrm{eV}^{2}$ and the atmospheric squared-mass difference $\Delta m_{\text {ATM }}^{2}=\left|\Delta m_{31}^{2}\right| \simeq\left|\Delta m_{32}^{2}\right| \approx 2.5 \times 10^{-3} \mathrm{eV}^{2}$, which generate the observed solar, atmospheric and long-baseline neutrino oscillations explained by the standard three-neutrino mixing (see refs. $[18,19]$ ). The $3+1$ active-sterile mixing scheme is a perturbation of the standard three-neutrino mixing in which the $3 \times 3$ unitary mixing matrix $U$ is extended to a $4 \times 4$ unitary mixing matrix with $\left|U_{e 4}\right|^{2},\left|U_{\mu 4}\right|^{2},\left|U_{\tau 4}\right|^{2} \ll 1$. The effective oscillation probabilities of the flavor neutrinos in short-baseline experiments are given by [20]

$$
P_{\alpha \beta}^{(\mathrm{SBL})} \simeq\left|\delta_{\alpha \beta}-\sin ^{2} 2 \vartheta_{\alpha \beta} \sin ^{2}\left(\frac{\Delta m_{41}^{2} L}{4 E}\right)\right|,
$$

where $\alpha, \beta=e, \mu, \tau, s, L$ is the source-detector distance and $E$ is the neutrino energy. The short-baseline oscillation amplitudes depend only on the absolute values of the elements in the fourth column of the mixing matrix:

$$
\sin ^{2} 2 \vartheta_{\alpha \beta}=\left.4\left|U_{\alpha 4}\right|^{2}\left|\delta_{\alpha \beta}-\right| U_{\beta 4}\right|^{2} \mid .
$$

Hence, the transition probabilities of neutrinos and antineutrinos are equal and it is not possible to measure in short-baseline experiments any $\mathrm{CP}$-violating effect generated by the complex phases in the mixing matrix. ${ }^{1}$

In this paper we update the analysis of short-baseline neutrino oscillation data $[7$, $12,32,33]$ revising the analysis of the rates measured in reactor neutrino experiments according to ref. [34] and taking into account the recent measurements of the MINOS [35], IceCube [36], and NEOS [37] experiments. The MINOS and IceCube constraints on $\nu_{\mu}$ and $\bar{\nu}_{\mu}$ disappearance are expected [38] to disfavor the low- $\Delta m_{41}^{2}-$ high- $\sin ^{2} 2 \vartheta_{\mu \mu}$ and the low- $\Delta m_{41}^{2}$-high- $\sin ^{2} 2 \vartheta_{e \mu}$ parts of the allowed region that we found in our previous analyses $[7,12,32,33]$, as was found in the $3+1$ global fit presented in ref. [39], which updated ref. [40] with the addition of the IceCube data. The NEOS [37] collaboration measured the spectrum of reactor $\bar{\nu}_{e}$ 's at a distance of $24 \mathrm{~m}$ and normalized their data to the Daya Bay spectrum [41] measured at the large distance of about $550 \mathrm{~m}$, where short-baseline oscillations are averaged out. Analyzing this normalized spectrum with short-baseline oscillations they found the best fit at $\Delta m_{41}^{2}=1.73 \mathrm{eV}^{2}$ and $\sin ^{2} 2 \vartheta_{e e}=0.05$, with a $\chi^{2}$ which is lower by 6.5 with respect to the standard case of three-neutrino mixing without shortbaseline oscillations. This is a $2.1 \sigma$ indication in favor of short-baseline oscillations and it is intriguing to note that the best-fit value of $\Delta m_{41}^{2}$ is close to the best-fit value found in our previous global analysis of short-baseline data [12], $\Delta m_{41}^{2}=1.6 \mathrm{eV}^{2}$, albeit with a larger $\sin ^{2} 2 \vartheta_{e e}=0.11$. However, as one can see from table 5 of ref. [12], the lower bound of the $3 \sigma$ allowed range of $\sin ^{2} 2 \vartheta_{e e}$ was 0.046 , which is below the NEOS best-fit value. Hence, the NEOS data are not incompatible with the global indications of short-baseline oscillations and we expect that their inclusion in the fit will lead to a shift of the allowed region towards smaller values of $\sin ^{2} 2 \vartheta_{e e}$ and, consequently, of $\left|U_{e 4}\right|^{2}$.

It is well known $[11,12,20,33,42-49]$ that the global fits of short-baseline data are affected by the so-called "appearance-disappearance" tension which is present [17] for any

\footnotetext{
${ }^{1} \mathrm{CP}$ violating effects due to active-sterile neutrino mixing can, however, be observed in long-baseline [2130] and solar [31] neutrino experiments.
} 
number $N_{s}$ of sterile neutrinos in $3+N_{s}$ mixing schemes which are perturbations of the standard three-neutrino mixing required for the explanation of the observation of solar, atmospheric and long-baseline neutrino oscillations (see refs. $[18,19]$ ). We expect that the inclusion in the global fit of the recent measurements of the MINOS [35], IceCube [36], and NEOS [37] experiment will increase somewhat the appearance-disappearance tension. In ref. [33] we proposed a "pragmatic approach" in which the appearance-disappearance tension is alleviated by excluding from the global fit the low-energy bins of the MiniBooNE experiment $[50,51]$ which have an anomalous excess of $\nu_{e}$-like events that is under investigation in the MicroBooNE experiment at Fermilab [52]. In this paper we will discuss the effect of MINOS, IceCube and NEOS data on the appearance-disappearance tension and how much it is alleviated in the pragmatic approach.

The plan of the paper is as follows. In section 2 we consider the experimental data on short-baseline $\nu_{e}$ and $\bar{\nu}_{e}$ disappearance, motivated by the Gallium and reactor anomalies. In section 3 we consider the global fit of appearance and disappearance data, which is motivated by the addition of the LSND anomaly to the Gallium and reactor anomalies. We draw our conclusions in section 4 .

\section{$2 \quad \nu_{e}$ and $\bar{\nu}_{e}$ disappearance}

In this section we consider only the experimental data on short-baseline $\nu_{e}$ and $\bar{\nu}_{e}$ disappearance, which include the Gallium neutrino anomaly [3-7] and the reactor antineutrino anomaly [8]. First, we discuss in subsection 2.1 our evaluation of the reactor antineutrino anomaly by considering the updated results of the reactor $\bar{\nu}_{e}$ rates measured in several reactor neutrino experiments. In subsection 2.2 we add the constraints of the spectra measured in the old Bugey-3 experiment [53] and in the recent NEOS experiment [37]. Finally, in subsection 2.3 we present our results for the combined fit of reactor and Gallium data and for the global fit of all the $\nu_{e}$ and $\bar{\nu}_{e}$ disappearance data.

\subsection{Reactor rates}

The reactor neutrino experiments which measured the absolute antineutrino flux that are considered in our analysis ${ }^{2}$ are listed in table 1 . For each experiment labeled with the index $a$, we listed the corresponding four fission fractions $f_{k}^{a}$, the ratio of measured and predicted rates $R_{a}^{\exp }$, the corresponding relative experimental uncertainty $\sigma_{a}^{\exp }$, the relative uncertainty $\sigma_{a}^{\text {cor }}$ which is correlated in each group of experiments indicated by the braces, and the relative theoretical uncertainty $\sigma_{a}^{\text {the }}$ which is correlated among all the experiments. The ratios $R_{a}^{\exp }$ and the uncertainties $\sigma_{a}^{\exp }$ and $\sigma_{a}^{\text {cor }}$ are the same as those in ref. [34]. In the following we repeat for convenience ${ }^{3}$ their derivation and we explain the derivation of the relative theoretical uncertainty $\sigma_{a}^{\text {the }}$.

The ratios of measured and predicted rates of the short-baseline experiments Bugey4 [55], Rovno91 [56], Bugey-3 [53], Gosgen [57], ILL [58, 59], Krasnoyarsk87 [60], Krasnoyarsk94 [61, 62], Rovno88 [63], and SRP [64] have been calculated by the Saclay group in

\footnotetext{
${ }^{2}$ We do not consider the still preliminary data of the Neutrino-4 experiment [54].

${ }^{3}$ We also correct, in table 1 , the misprints of the Rovno 88 correlations in table 2 of ref. [34].
} 


\begin{tabular}{|c|c|c|c|c|c|c|c|c|c|c|}
\hline$a$ & Experiment & $f_{235}^{a}$ & $f_{238}^{a}$ & $f_{239}^{a}$ & $f_{241}^{a}$ & $R_{a}^{\exp }$ & $\sigma_{a}^{\exp }[\%]$ & $\sigma_{a}^{\text {cor }}[\%]$ & $\sigma_{a}^{\text {the }}[\%]$ & $L_{a}[\mathrm{~m}]$ \\
\hline 1 & Bugey-4 & 0.538 & 0.078 & 0.328 & 0.056 & 0.932 & 1.4 & \multirow{2}{*}{1.4} & 2.5 & 15 \\
\hline 2 & Rovno91 & 0.606 & 0.074 & 0.277 & 0.043 & 0.930 & 2.8 & & 2.4 & 18 \\
\hline 3 & Rovno88-1I & 0.607 & 0.074 & 0.277 & 0.042 & 0.907 & 6.4 & \multirow{2}{*}{3.1} & 2.4 & 18 \\
\hline 4 & Rovno88-2I & 0.603 & 0.076 & 0.276 & 0.045 & 0.938 & 6.4 & & 2.4 & 18 \\
\hline 5 & Rovno88-1S & 0.606 & 0.074 & 0.277 & 0.043 & 0.962 & 7.3 & \multirow{3}{*}{3.1} & 2.4 & 18 \\
\hline 6 & Rovno88-2S & 0.557 & 0.076 & 0.313 & 0.054 & 0.949 & 7.3 & & 2.5 & 25 \\
\hline 7 & Rovno88-2S & 0.606 & 0.074 & 0.274 & 0.046 & 0.928 & 6.8 & & 2.4 & 18 \\
\hline 8 & Bugey-3-15 & 0.538 & 0.078 & 0.328 & 0.056 & 0.936 & 4.2 & \multirow{3}{*}{4.0} & 2.5 & 15 \\
\hline 9 & Bugey-3-40 & 0.538 & 0.078 & 0.328 & 0.056 & 0.942 & 4.3 & & 2.5 & 40 \\
\hline 10 & Bugey-3-95 & 0.538 & 0.078 & 0.328 & 0.056 & 0.867 & 15.2 & & 2.5 & 95 \\
\hline 11 & Gosgen-38 & 0.619 & 0.067 & 0.272 & 0.042 & 0.955 & 5.4 & \multirow{4}{*}{2.0} & 2.4 & 37.9 \\
\hline 12 & Gosgen-46 & 0.584 & 0.068 & 0.298 & 0.050 & 0.981 & 5.4 & & 2.4 & 45.9 \\
\hline 13 & Gosgen-65 & 0.543 & 0.070 & 0.329 & 0.058 & 0.915 & 6.7 & & 2.4 & 64.7 \\
\hline 14 & ILL & 1 & 0 & 0 & 0 & 0.792 & 9.1 & & 2.4 & 8.76 \\
\hline 15 & Krasnoyarsk87-33 & 1 & 0 & 0 & 0 & 0.925 & 5.0 & \multirow{2}{*}{4.1} & 2.4 & 32.8 \\
\hline 16 & Krasnoyarsk87-92 & 1 & 0 & 0 & 0 & 0.942 & 20.4 & & 2.4 & 92.3 \\
\hline 17 & Krasnoyarsk94-57 & 1 & 0 & 0 & 0 & 0.936 & 4.2 & 0 & 2.4 & 57 \\
\hline 18 & Krasnoyarsk99-34 & 1 & 0 & 0 & 0 & 0.946 & 3.0 & 0 & 2.4 & 34 \\
\hline 19 & SRP-18 & 1 & 0 & 0 & 0 & 0.941 & 2.8 & 0 & 2.4 & 18.2 \\
\hline 20 & SRP-24 & 1 & 0 & 0 & 0 & 1.006 & 2.9 & 0 & 2.4 & 23.8 \\
\hline 21 & Nucifer & 0.926 & 0.061 & 0.008 & 0.005 & 1.014 & 10.7 & 0 & 2.3 & 7.2 \\
\hline 22 & Chooz & 0.496 & 0.087 & 0.351 & 0.066 & 0.996 & 3.2 & 0 & 2.5 & $\approx 1000$ \\
\hline 23 & Palo Verde & 0.600 & 0.070 & 0.270 & 0.060 & 0.997 & 5.4 & 0 & 2.4 & $\approx 800$ \\
\hline 24 & Daya Bay & 0.561 & 0.076 & 0.307 & 0.056 & 0.946 & 2.0 & 0 & 2.5 & $\approx 550$ \\
\hline 25 & RENO & 0.569 & 0.073 & 0.301 & 0.056 & 0.944 & 2.2 & 0 & 2.4 & $\approx 411$ \\
\hline 26 & Double Chooz & 0.511 & 0.087 & 0.340 & 0.062 & 0.935 & 1.4 & 0 & 2.5 & $\approx 415$ \\
\hline
\end{tabular}

Table 1. List of the experiments which measured the absolute reactor antineutrino flux. For each experiment numbered with the index $a$, the index $k=235,238,239,241$ indicate the four fissionable isotopes ${ }^{235} \mathrm{U},{ }^{238} \mathrm{U},{ }^{239} \mathrm{Pu}$, and ${ }^{241} \mathrm{Pu}, f_{k}^{a}$ are the fission fractions, $R_{a}^{\exp }$ is the ratio of measured and predicted rates, $\sigma_{a}^{\exp }$ is the corresponding relative experimental uncertainty, $\sigma_{a}^{\text {cor }}$ is the relative systematic uncertainty which is correlated in each group of experiments indicated by the braces, $\sigma_{a}^{\text {the }}$ is the relative theoretical uncertainty which is correlated among all the experiments, and $L_{a}$ is the source-detector distance.

ref. [8]. The calculation of the ${ }^{235} \mathrm{U},{ }^{239} \mathrm{Pu}$, and ${ }^{241} \mathrm{Pu}$ antineutrino fluxes was subsequently improved by P. Huber in [65]. We took into account this correction with the following rescaling of the Saclay ratios: ${ }^{4}$

$$
R_{a}^{\exp }=R_{a, \mathrm{~S}}^{\exp } \frac{\sum_{k} f_{k}^{a} \sigma_{f, k}^{\mathrm{S}}}{\sum_{k} f_{k}^{a} \sigma_{f, k}^{\mathrm{SH}}} \quad(a=1, \ldots, 17,19,20),
$$

where $\sigma_{f, k}^{\mathrm{S}}$ and $\sigma_{f, k}^{\mathrm{SH}}$ are, respectively, the Saclay [8] and Saclay+Huber [65] cross sections

\footnotetext{
${ }^{4}$ The missing index $a=18$ corresponds to the Krasnoyarsk99-34 experiment discussed below.
} 


\begin{tabular}{|crr|}
\hline$k$ & \multicolumn{1}{c}{$\sigma_{f, k}^{\mathrm{S}}$} & \multicolumn{1}{c|}{$\sigma_{f, k}^{\mathrm{SH}}$} \\
\hline 235 & $6.61 \pm 2.11 \%$ & $6.69 \pm 2.44 \%$ \\
238 & $10.10 \pm 8.15 \%$ & $10.10 \pm 8.15 \%$ \\
239 & $4.34 \pm 2.45 \%$ & $4.40 \pm 2.88 \%$ \\
241 & $5.97 \pm 2.15 \%$ & $6.03 \pm 2.60 \%$ \\
\hline
\end{tabular}

Table 2. Cross sections per fission of the four fissionable isotopes calculated by the Saclay (S) group $\left(\sigma_{f, k}^{\mathrm{S}}\right)$ in ref. [8] and those obtained from the Huber $(\mathrm{SH})$ correction $\left(\sigma_{f, k}^{\mathrm{SH}}\right)$ in ref. [65]. The units are $10^{-43} \mathrm{~cm}^{2} /$ fission. The index $k=235,238,239,241$ indicates the four isotopes ${ }^{235} \mathrm{U},{ }^{238} \mathrm{U}$, ${ }^{239} \mathrm{Pu}$, and ${ }^{241} \mathrm{Pu}$.

per fission given in table 2. The index $k=235,238,239,241$ indicates the four fissionable isotopes ${ }^{235} \mathrm{U},{ }^{238} \mathrm{U},{ }^{239} \mathrm{Pu}$, and ${ }^{241} \mathrm{Pu}$ which constitute the reactor fuel.

We considered the Krasnoyarsk99-34 experiment [66] that was not considered in refs. [8, 67], by rescaling the value of the corresponding experimental cross section per fission in comparison with the Krasnoyarsk94-57 result. For the long-baseline experiments Chooz [68] and Palo Verde [69], we applied the rescaling in eq. (2.1) with the ratios $R_{a, \mathrm{~S}}^{\exp }$ given in ref. [67], divided by the corresponding survival probability $P_{\text {sur }}$ caused by $\vartheta_{13}$. For Nucifer [70], Daya Bay [41], RENO [71], and Double Chooz $^{5}$ we use the ratios provided by the respective experimental collaborations.

The experimental uncertainties and their correlations listed in table 1 have been obtained from the corresponding experimental papers. In particular:

- The Bugey-4 and Rovno91 experiments have a correlated 1.4\% uncertainty, because they used the same detector [55].

- The Rovno88 experiments have a correlated $2.2 \%$ reactor-related uncertainty [63]. In addition, each of the two groups of integral (Rovno88-1I and Rovno88-2I) and spectral (Rovno88-1S, Rovno88-2S, and Rovno88-3S) measurements have a correlated 3.1\% detector-related uncertainty [63].

- The Bugey-3 experiments have a correlated $4.0 \%$ uncertainty obtained from table 9 of [55].

- The Gosgen and ILL experiments have a correlated 3.8\% uncertainty, because they used the same detector [57]. In addition, the Gosgen experiments have a correlated $2.0 \%$ reactor-related uncertainty [57].

- The 1987 Krasnoyarsk87-33 and Krasnoyarsk87-92 experiments have a correlated $4.1 \%$ uncertainty, because they used the same detector at 32.8 and $92.3 \mathrm{~m}$ from two reactors [60]. The Krasnoyarsk94-57 experiment was performed in 1990-94 with a different detector at 57.0 and $57.6 \mathrm{~m}$ from the same two reactors [61]. The Krasnoyarsk99-34 experiment was performed in 1997-99 with a new integral-type

\footnotetext{
${ }^{5}$ Double Chooz Collaboration, Private Communication.
} 


\begin{tabular}{|c|llll|}
\hline & ${ }^{235} \mathrm{U}$ & ${ }^{238} \mathrm{U}$ & ${ }^{239} \mathrm{Pu}$ & ${ }^{241} \mathrm{Pu}$ \\
\hline${ }^{235} \mathrm{U}$ & 0.0267 & 0 & 0.0203 & 0.0255 \\
${ }^{238} \mathrm{U}$ & 0 & 0.6776 & 0 & 0 \\
${ }^{239} \mathrm{Pu}$ & 0.0203 & 0 & 0.0161 & 0.0194 \\
${ }^{241} \mathrm{Pu}$ & 0.0255 & 0 & 0.0194 & 0.0246 \\
\hline
\end{tabular}

Table 3. Covariance matrix of the cross sections per fission of the four fissionable isotopes.

detector at $34 \mathrm{~m}$ from the same reactor of the Krasnoyarsk87-33 experiment [72]. There may be reactor-related uncertainties correlated among the four Krasnoyarsk experiments, but, taking into account the time separations and the absence of any information, we conservatively neglected them.

- Following ref. [67], we considered the two SRP measurements as uncorrelated, because the two measurements would be incompatible with the correlated uncertainty estimated in ref. [64].

For each experiment labeled with the index $a$, the relative theoretical uncertainty $\sigma_{a}^{\text {the }}$ in table 1 is given by

$$
\sigma_{a}^{\text {the }}=\frac{\sqrt{\sum_{k, j} f_{k}^{a} \rho_{k j}^{\mathrm{SH}} f_{j}^{a}}}{\sum_{k} f_{k}^{a} \sigma_{f, k}^{\mathrm{SH}}},
$$

where $\rho^{\mathrm{SH}}$ is the covariance matrix of the cross sections per fission of the four fissionable isotopes given in table 3. In this covariance matrix, $\sigma_{f, 238}^{\mathrm{SH}}$ is uncorrelated from the other cross sections per fission and the corresponding uncertainty is that given in ref. [8] (we neglected the correlation due to the cross section uncertainty, which is of the order of $0.1 \%$ ). The other three cross sections per fission have been calculated using the Huber [65] antineutrino fluxes which have been obtained by inverting the spectra of the electrons emitted by the $\beta$ decays of the products of the thermal fission of ${ }^{235} \mathrm{U},{ }^{239} \mathrm{Pu}$, and ${ }^{241} \mathrm{Pu}$ which have been measured at ILL in the 80's [73-75]. As explained in ref. [65], the values of the three antineutrino fluxes are correlated. We calculated the uncertainties and correlations of $\sigma_{f, 235}^{\mathrm{SH}}, \sigma_{f, 239}^{\mathrm{SH}}$, and $\sigma_{f, 241}^{\mathrm{SH}}$ using the information given in ref. [65]. The square roots of the diagonal elements of the covariance matrix $\rho^{\mathrm{SH}}$ in table 3 give the uncertainties of the cross sections per fission reported in table 2. One can see that the uncertainties of $\sigma_{f, 235}^{\mathrm{SH}}$, $\sigma_{f, 239}^{\mathrm{SH}}$, and $\sigma_{f, 241}^{\mathrm{SH}}$ are slightly larger than those calculated by Saclay group in ref. [8].

Let us note that after the discovery of the unexpected " $5 \mathrm{MeV}$ bump" in the spectrum of the RENO [76], Double Chooz [77], and Daya Bay [78] experiments it is believed [79, 80] that the theoretical uncertainties of the reactor antineutrino fluxes may be larger than those calculated in refs. [65, 81]. However, since there is no well-motivated quantitative estimation of how much the theoretical uncertainties should be increased, we are compelled to use the uncertainties calculated in refs. [65, 81]. 


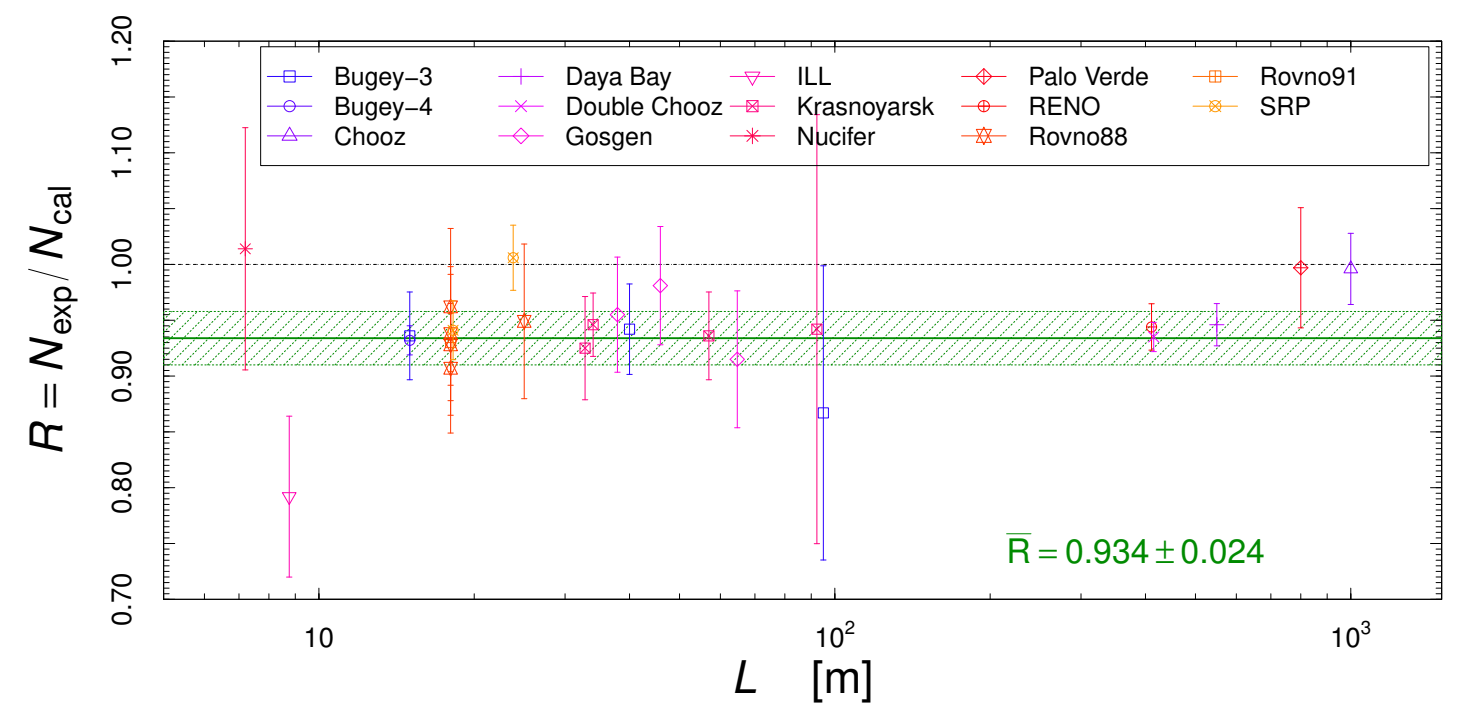

Figure 1. Ratios $R$ of the reactor experiments considered in our analysis as functions of the reactor-detector distance $L$. The horizontal band shows the average ratio $\bar{R}$ and its uncertainty. The error bars show the experimental uncertainties.

Figure 1 shows the experimental ratios as functions of the reactor-detector distance $L$. The horizontal band shows the average ratio $\bar{R}$ and its uncertainty,

$$
\bar{R}=0.934 \pm 0.024,
$$

which has been obtained by summing in quadrature the experimental and theoretical uncertainties. Hence, the reactor antineutrino anomaly is at the level of about $2.8 \sigma$.

The slight difference of the value of $\bar{R}$ in eq. (2.3) with respect to our previous estimates in refs. $[7,12]$ is due to the following three changes in our analysis:

1. The revaluation [34] of the experimental ratios $R_{a}^{\exp }$ listed in table 1 .

2. The new treatment of the theoretical uncertainties $\sigma_{a}^{\text {the }}$ according to eq. (2.2) instead of considering an unrealistic common $2.0 \%$ [8].

3. The new data of the Nucifer, Daya Bay, RENO and Double Chooz experiments and the consideration for the first time of the Krasnoyarsk99-34 experiment.

The reactor antineutrino anomaly can be explained in the framework of $3+1$ neutrino mixing through neutrino oscillations generated by the effective mixing angle $\sin ^{2} 2 \vartheta_{e e}=$ $4\left|U_{e 4}\right|^{2}\left(1-\left|U_{e 4}\right|^{2}\right)$, which determines the survival probability of $\nu_{e}$ 's and $\bar{\nu}_{e}$ 's according to eq. (1.1). The result of the fit of the reactor rates are given in the first column of table 4 and in figure 2(a), where we have drawn the allowed regions in the $\sin ^{2} 2 \vartheta_{e e}-\Delta m_{41}^{2}$ plane.

From figure 2(a) one can see that the allowed $1 \sigma$ region $^{6}$ in the $\sin ^{2} 2 \vartheta_{e e}-\Delta m_{41}^{2}$ plane is at a rather low value of $\Delta m_{41}^{2}$, but the allowed regions at $2 \sigma$ and $3 \sigma$ extend to higher

\footnotetext{
${ }^{6}$ In all the paper we consider allowed regions at $1 \sigma, 2 \sigma$, and $3 \sigma$, which correspond, respectively, to $68.27 \%, 95.45 \%$, and $99.73 \%$ confidence level. The allowed regions in two-parameter planes are drawn considering two degrees of freedom, which correspond, respectively, to $\Delta \chi^{2}=2.30,6.18$, and 11.83 from the minimum $\chi_{\min }^{2}$.
} 


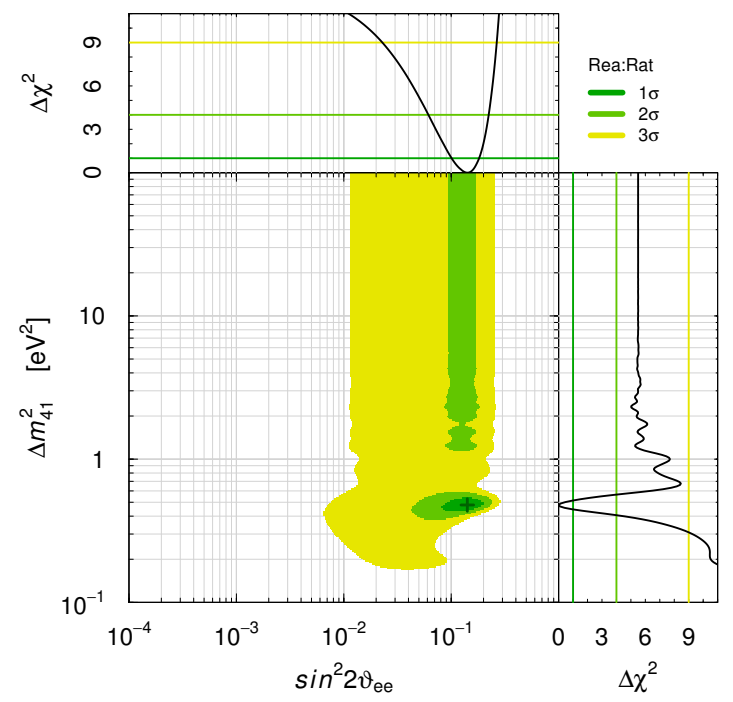

(a)

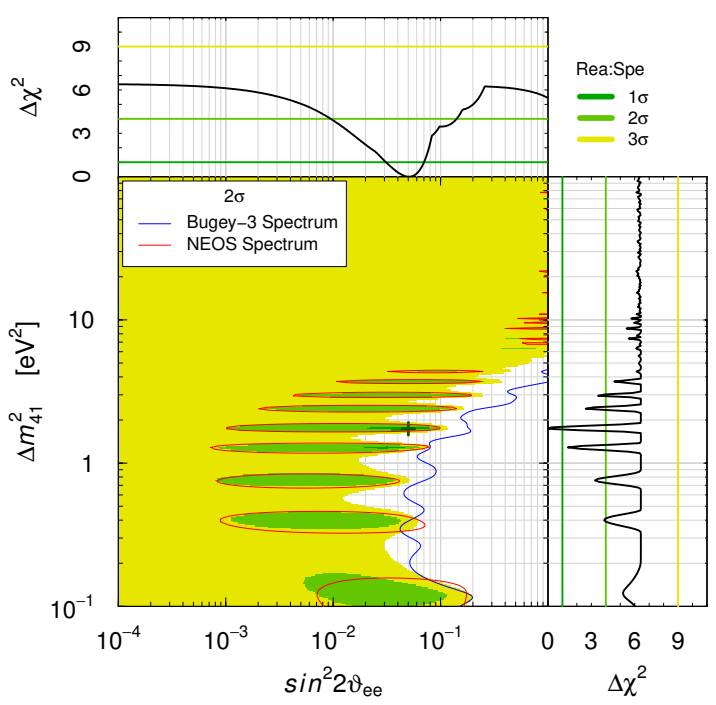

(b)

Figure 2. Allowed regions in the $\sin ^{2} 2 \vartheta_{e e^{-}} \Delta m_{41}^{2}$ plane and marginal $\Delta \chi^{2}$ 's for $\sin ^{2} 2 \vartheta_{e e}$ and $\Delta m_{41}^{2}$ obtained from: (a) the combined fit of the rates of the reactor neutrino experiments in table 1; (b) the combined fit of the spectra of Bugey-3 [53] and NEOS [37] reactor antineutrino experiments; the best-fit points corresponding to $\chi_{\min }^{2}$ in table 4 are indicated by crosses.

\begin{tabular}{|c|cccccc|}
\hline & Rea:Rat & Rea:Spe & Rea:Rat+Spe & Rea+Gal & $\nu_{e}$ Dis & $\nu_{e}$ Dis $\beta$ \\
\hline$\chi_{\min }^{2}$ & 12.4 & 73.9 & 94.6 & 107.1 & 163.0 & 163.1 \\
$\mathrm{NDF}$ & 24 & 82 & 108 & 112 & 174 & 176 \\
$\mathrm{GoF}$ & $100 \%$ & $73 \%$ & $82 \%$ & $61 \%$ & $71 \%$ & $75 \%$ \\
$\Delta m_{41}^{2}$ & 0.48 & 1.7 & 1.7 & 3.0 & 1.7 & 1.7 \\
$\sin ^{2} 2 \vartheta_{e e}$ & 0.14 & 0.050 & 0.062 & 0.14 & 0.066 & 0.066 \\
$\left|U_{e 4}\right|^{2}$ & 0.037 & 0.013 & 0.016 & 0.036 & 0.017 & 0.017 \\
$\Delta \chi_{\mathrm{NO}}^{2}$ & 13.1 & 6.4 & 11.3 & 16.0 & 14.1 & 14.0 \\
$n \sigma_{\mathrm{NO}}$ & 3.2 & 2.1 & 2.9 & 3.6 & 3.3 & 3.3 \\
\hline
\end{tabular}

Table 4. Results of the fits of $\nu_{e}$ and $\bar{\nu}_{e}$ disappearance data: minimum $\chi^{2}\left(\chi_{\min }^{2}\right)$, number of degrees of freedom (NDF), goodness of fit (GoF), best fit values of $\Delta m_{41}^{2}, \sin ^{2} 2 \vartheta_{e e}$, and $\left|U_{e 4}\right|^{2}, \chi^{2}$ difference $\Delta \chi_{\mathrm{NO}}^{2}$ between the $\chi^{2}$ of no oscillations and $\chi_{\mathrm{min}}^{2}$, and the resulting number of $\sigma^{\prime} \mathrm{s}\left(n \sigma_{\mathrm{NO}}\right)$ for two degrees of freedom corresponding to two fitted parameters $\left(\Delta m_{41}^{2}\right.$ and $\left.\sin ^{2} 2 \vartheta_{e e}\right)$. The columns correspond to the fits of the data of reactor rates (Rea:Rat), reactor spectra (Rea:Spe), reactor rates and spectra (Rea:Rat+Spe), reactor and Gallium data (Rea+Gal), $\nu_{e}$ and $\bar{\nu}_{e}$ disappearance data $\left(\nu_{e}\right.$ Dis $), \nu_{e}$ and $\bar{\nu}_{e}$ disappearance data and $\beta$ decay constraints $\left(\nu_{e}\right.$ Dis $\left.+\beta\right)$.

values of $\Delta m_{41}^{2}$, without an upper bound. The favorite values of the amplitude $\sin ^{2} 2 \vartheta_{e e}$ of $\nu_{e}$-disappearance oscillations are around 0.1 , but the allowed $3 \sigma$ region in the $\sin ^{2} 2 \vartheta_{e e^{-}}$ $\Delta m_{41}^{2}$ plane covers the range $0.0066 \lesssim \sin ^{2} 2 \vartheta_{e e} \lesssim 0.28$, which corresponds to $0.0017 \lesssim$ $\left|U_{e 4}\right|^{2} \lesssim 0.076$. 
Table 4 gives the $\chi^{2}$ difference $\Delta \chi_{\text {NO }}^{2}$ between the $\chi^{2}$ of no oscillations and $\chi_{\min }^{2}$, and the corresponding number of $\sigma$ 's for the two degrees of freedom corresponding to the two fitted parameters $\Delta m_{41}^{2}$ and $\sin ^{2} 2 \vartheta_{e e}$. The case of no oscillations turns out to be disfavored at the level of $3.2 \sigma$.

\section{$2.2 \quad$ Reactor spectra}

In our previous analyses $[7,12,32,33]$ we considered the ratio of the spectra measured at $40 \mathrm{~m}$ and $15 \mathrm{~m}$ from the source in the Bugey-3 experiment [53]. These data provide robust information on short-baseline $\bar{\nu}_{e}$ disappearance, which is independent of any theoretical calculation of the spectrum and of the solution of the " $5 \mathrm{MeV}$ bump" problem mentioned in subsection 2.1.

In this paper we add the constraints obtained in the recent NEOS experiment by taking into account the $\chi^{2}$ corresponding to figure 4 of ref. [37], which has been kindly provided to us by the NEOS Collaboration. ${ }^{7}$ The NEOS constraints are mostly independent of theoretical calculations of the spectrum and of the solution of the "5 MeV bump" problem, because the NEOS $\chi^{2}$ has been obtained by fitting the NEOS spectrum normalized to the Daya Bay spectrum [41] measured at the large distance of about $550 \mathrm{~m}$, where the shortbaseline oscillations due to $\Delta m_{41}^{2}$ are averaged out. A small dependence on the theoretical calculation of the spectrum $[65,81]$ comes from the corrections due to the differences of the fission fractions of the NEOS and Daya Bay reactors [37, 41] and a small dependence on the "5 MeV bump" problem comes from a possible dependence of the " $5 \mathrm{MeV}$ bump" on the different fission fractions of NEOS and Daya Bay [82]. We neglect these possible small effects.

The results of the fit of the Bugey-3 and NEOS spectra are given in the second column of table 4 and in figure 2(b), where one can see that the NEOS constraints are dominating. There are closed allowed islands at $2 \sigma$ which are determined mainly by the NEOS data and the best-fit values of the oscillation parameters in table 4 correspond to the best fit reported in ref. [37]. Hence, the NEOS constraints can be interpreted as a weak indication in favor of short-baseline oscillations which may be compatible with the reactor antineutrino anomaly based on the reactor rates discussed in subsection 2.1. This is confirmed by the disfavoring of the case of no oscillations at the level of $2.1 \sigma$, as shown in table 4 .

The third column of table 4 and figure 3(a) show the results of the combined fit of the rate and spectral data of reactor antineutrino experiments. As reported in table 4, the combined fit disfavors the case of no oscillations at the level of $2.9 \sigma$, which is about the same level obtained from the analysis of the reactor rates alone. Hence, the NEOS constraints do not exclude the reactor antineutrino anomaly. However, in spite of the weak NEOS indication in favor of short-baseline oscillations discussed above, the statistical significance of the anomaly does not increase by including the NEOS data because there is a mild tension with the reactor rates which is illustrated by the $2 \sigma$ contours in figure $3(\mathrm{a})$. Indeed, the rates-spectra parameter goodness-of-fit is only $2 \%\left(\Delta \chi^{2} / \mathrm{NDF}=8.3 / 2\right)$.

\footnotetext{
${ }^{7}$ NEOS Collaboration, Private Communication.
} 


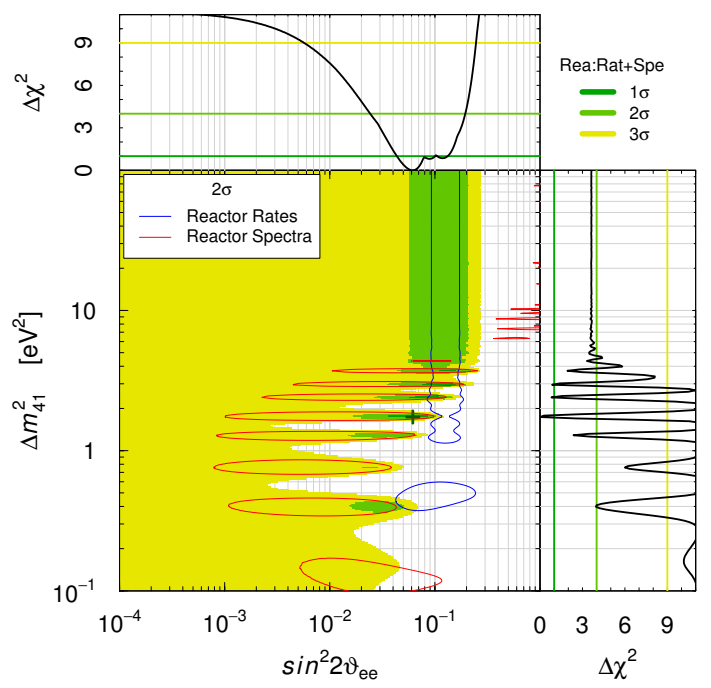

(a)

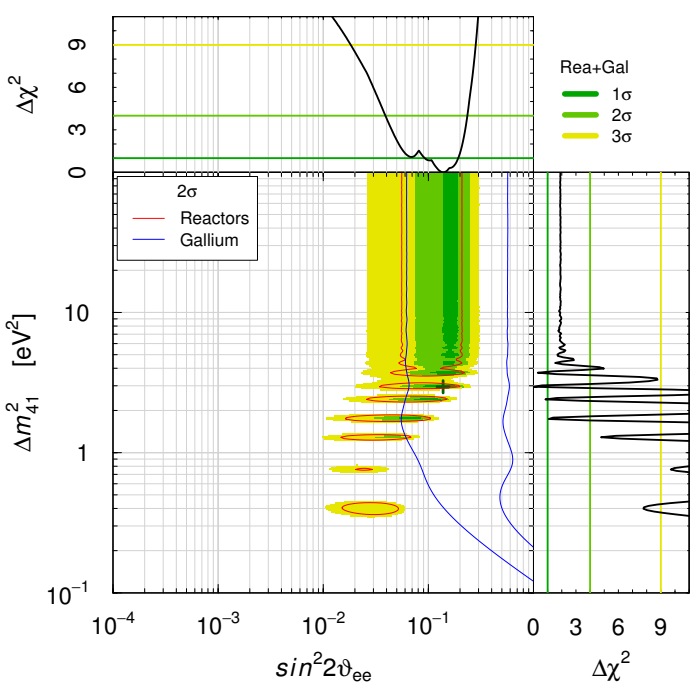

(b)

Figure 3. Allowed regions in the $\sin ^{2} 2 \vartheta_{e e}-\Delta m_{41}^{2}$ plane and marginal $\Delta \chi^{2}$ 's for $\sin ^{2} 2 \vartheta_{e e}$ and $\Delta m_{41}^{2}$ obtained from: (a) the combined fit of the rate and spectral data of reactor antineutrino experiments; (b) the combined fit of the reactor and Gallium data. The best-fit points corresponding to $\chi_{\min }^{2}$ in table 4 are indicated by crosses.

\subsection{Global $\nu_{e}$ and $\bar{\nu}_{e}$ disappearance}

In this subsection we discuss the combination of the reactor data with the data of the Gallium neutrino anomaly, other $\nu_{e}$ and $\bar{\nu}_{e}$ disappearance data and the $\beta$-decay constraints of the Mainz [83] and Troitsk [84, 85] experiments.

The fourth column of table 4 and figure $3(\mathrm{~b})$ show the results of the combined fit of reactor and Gallium data. Since both sets of data indicate short-baseline $\nu_{e}$ and $\bar{\nu}_{e}$ disappearance, the statistical significance of active-sterile neutrino oscillations increases to $3.6 \sigma$ and the $3 \sigma$ allowed regions in the $\sin ^{2} 2 \vartheta_{e e}-\Delta m_{41}^{2}$ plane are confined to $0.010 \lesssim$ $\sin ^{2} 2 \vartheta_{e e} \lesssim 0.30$ and $\Delta m_{41}^{2} \gtrsim 0.35 \mathrm{eV}^{2}$.

Besides the reactor and Gallium data, short-baseline $\nu_{e}$ disappearance ${ }^{8}$ is constrained by solar and KamLAND neutrino data [7, 87-90], by the KARMEN [91] and LSND [92] $\nu_{e}+{ }^{12} \mathrm{C} \rightarrow{ }^{12} \mathrm{~N}_{\text {g.s. }}+e^{-}$scattering data [46, 93] and by the T2K near detector constraints [94].

We updated our 2012 solar+KamLAND constraint [7] by including the latest solar data: the new results from the fourth phase of the Super-Kamiokande experiment [95] and the final results of Borexino phase-I [96]. We also updated the KamLAND data analysis by using the Saclay+Huber cross sections per fission [34]. Finally, we used the updated value of $\vartheta_{13}$ in the 2016 Review of Particle Physics [97]. We obtained the new marginal $\Delta \chi^{2}$ shown in figure 4 , where it is confronted with the old one obtained in ref. [7]. The new solar+KamLAND constraint is weaker than the 2012 one because of the larger Saclay+Huber reactor rate prediction used in the analysis of KamLAND data and because the new value of $\vartheta_{13}$ is smaller than that in 2012 .

\footnotetext{
${ }^{8}$ We work in the framework of a local quantum field theory in which the CPT symmetry implies that the survival probabilities of neutrinos and antineutrinos are equal (see ref. [86]).
} 


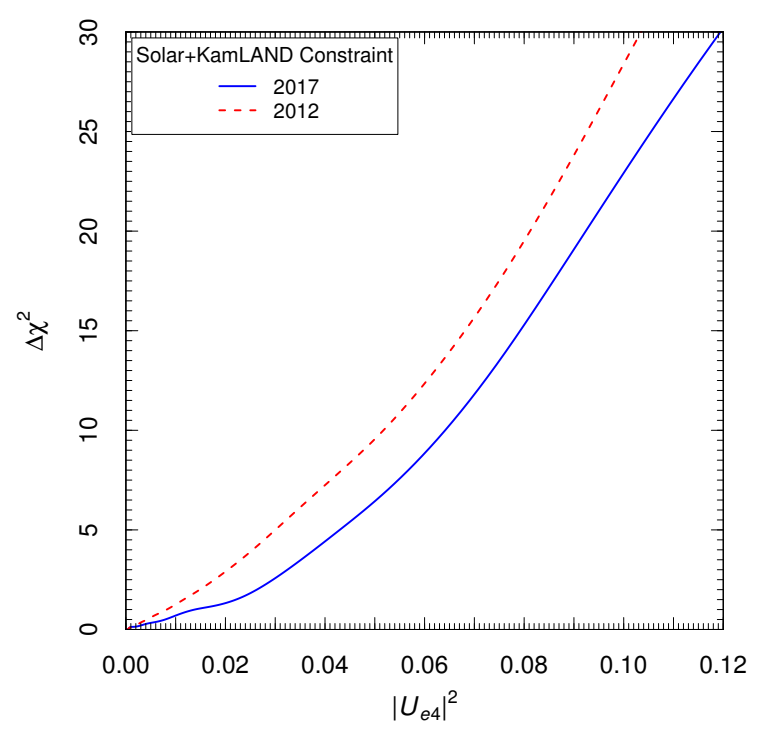

Figure 4. Marginal $\Delta \chi^{2}=\chi^{2}-\chi_{\min }^{2}$ as a function of $\sin ^{2} 2 \vartheta_{e e}$ obtained from the fit of current solar+KamLAND neutrino data (2017) compared with the one obtained in 2012 in ref. [7].

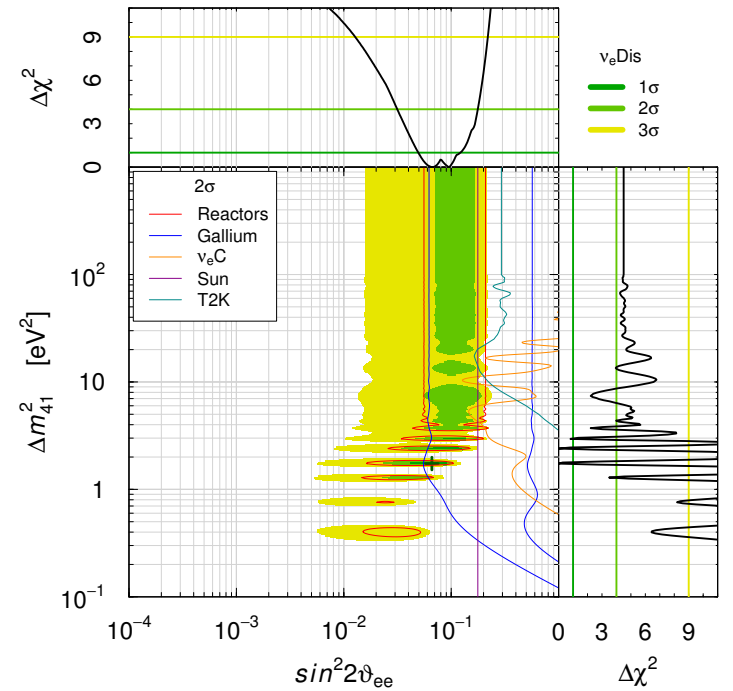

(a)

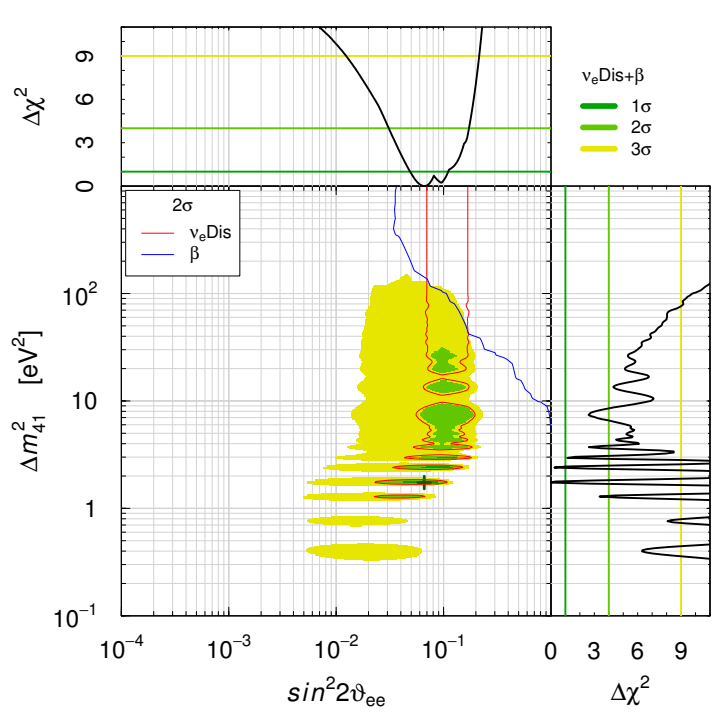

(b)

Figure 5. Allowed regions in the $\sin ^{2} 2 \vartheta_{e e}-\Delta m_{41}^{2}$ plane and marginal $\Delta \chi^{2}$ 's for $\sin ^{2} 2 \vartheta_{e e}$ and $\Delta m_{41}^{2}$ obtained from: (a) the combined fit of $\nu_{e}$ and $\bar{\nu}_{e}$ disappearance data; (b) the combined fit of $\nu_{e}$ and $\bar{\nu}_{e}$ disappearance data and the $\beta$-decay constraints of the Mainz [83] and Troitsk [84, 85] experiments. The best-fit points corresponding to $\chi_{\min }^{2}$ in table 4 are indicated by crosses.

The results of the combined analysis of all $\nu_{e}$ and $\bar{\nu}_{e}$ disappearance data are shown in the fifth column of table 4 and figure 5(a). Since the analysis of solar+KamLAND, $\nu_{e^{-}}{ }^{12} \mathrm{C}$, and T2K data do not show any indication of short-baseline $\nu_{e}$ disappearance, the combination with the reactor and Gallium data shifts the allowed regions in the $\sin ^{2} 2 \vartheta_{e e^{-}}$ $\Delta m_{41}^{2}$ plane in figure 5(a) to smaller values of $\sin ^{2} 2 \vartheta_{e e}$ with respect to figure $3(\mathrm{~b}): 0.0054 \lesssim$ $\sin ^{2} 2 \vartheta_{e e} \lesssim 0.23$. On the other hand, the $3 \sigma$ range of $\Delta m_{41}^{2}$ in figures $3(\mathrm{~b})$ and $5(\mathrm{a})$ is similar, with the lower bound $\Delta m_{41}^{2} \gtrsim 0.35 \mathrm{eV}^{2}$ and no upper bound. 
Large values of $\Delta m_{41}^{2}$ can be constrained with the data of $\beta$-decay experiments (see ref. [12]). As in ref. [32], we use the $\beta$-decay constraints of the Mainz [83] and Troitsk [84, 85] experiments, which give the allowed regions in the $\sin ^{2} 2 \vartheta_{e e}-\Delta m_{41}^{2}$ plane shown in figure $5(\mathrm{~b})$. One can see that the allowed regions are confined to the range

$$
1.3(0.33) \mathrm{eV}^{2} \lesssim \Delta m_{41}^{2} \lesssim 32(148) \mathrm{eV}^{2} \text { at } 2 \sigma(3 \sigma)
$$

For the oscillation length $L_{41}^{\text {osc }}=4 \pi E / \Delta m_{41}^{2}$ we have

$$
8(2) \mathrm{cm} \lesssim \frac{L_{41}^{\mathrm{osc}}}{E[\mathrm{MeV}]} \lesssim 2(7) \mathrm{m} \quad \text { at } \quad 2 \sigma(3 \sigma)
$$

This is a range of oscillation lengths which can be explored in a model independent way in the new short-baseline reactor neutrino experiments (DANSS [98], Neutrino-4 [54], PROSPECT [99], SoLid [100], STEREO [101]) and in the SOX [102] and BEST [103] radioactive source experiments by measuring the reactor antineutrino rate as a function of distance. However, they will need to be sensitive to small oscillations with the amplitude

$$
0.022(0.0050) \lesssim \sin ^{2} 2 \vartheta_{e e} \lesssim 0.19(0.23) \text { at } 2 \sigma(3 \sigma)
$$

Figure 6(a) shows the sensitivities of the short-baseline reactor antineutrino experiments DANSS [98], Neutrino-4 [104], PROSPECT [99], SoLid [105], and STEREO [106] in comparison with the allowed regions in the $\sin ^{2} 2 \vartheta_{e e}-\Delta m_{41}^{2}$ plane in figure 5 (b). One can see that they will cover most of the allowed regions for $\Delta m_{41}^{2} \lesssim 10 \mathrm{eV}^{2}$ and not too small $\sin ^{2} 2 \vartheta_{e e}$. Figure 6(b) shows the sensitivities of the CeSOX [102] and BEST [103] source experiments, of IsoDAR@KamLAND [107] and C-ADS [108], and of the KATRIN [109]) electron neutrino mass experiment. ${ }^{9}$ The source experiments will cover the large- $\sin ^{2} 2 \vartheta_{e e}$ parts of the allowed regions, the IsoDAR@KamLAND and C-ADS experiments can cover almost all the allowed regions, except the large- $\Delta m_{41}^{2}$ part and the small- $\sin ^{2} 2 \vartheta_{e e}$-small$\Delta m_{41}^{2}$ parts, and KATRIN will cover the large- $\Delta m_{41}^{2}$ part. Hence, there are favorable perspectives for a definitive solution of the short-baseline $\stackrel{(-)}{\nu}$ disappearance problem in the near future.

\section{$3 \quad$ Fits of appearance and disappearance data}

In this section we present the results of $3+1$ fits of short-baseline neutrino oscillation data which include $\nu_{\mu} \rightarrow \nu_{e}$ and $\bar{\nu}_{\mu} \rightarrow \bar{\nu}_{e}$ appearance data and $\nu_{\mu}$ and $\bar{\nu}_{\mu}$ disappearance data, in addition to the $\nu_{e}$ and $\bar{\nu}_{e}$ disappearance data considered in section 2. Our fits are based on a $\chi^{2}$ analysis in the four-dimensional space of the mixing parameters $\Delta m_{41}^{2},\left|U_{e 4}\right|^{2},\left|U_{\mu 4}\right|^{2}$, and $\left|U_{\tau 4}\right|^{2}$.

We consider the following short-baseline $\nu_{\mu} \rightarrow \nu_{e}$ and $\bar{\nu}_{\mu} \rightarrow \bar{\nu}_{e}$ appearance data: the LSND signal in favor of $\bar{\nu}_{\mu} \rightarrow \bar{\nu}_{e}$ transitions [1,2], the data of the MiniBooNE [50, 51]

\footnotetext{
${ }^{9}$ See also the studies in refs. [110-113]. There are also promising possibilities to observe the effects of eV-scale neutrinos in Holmium electron-capture experiments [114].
} 
experiment, and the constraints of the BNL-E776 [115], KARMEN [116], NOMAD [117], ICARUS [118] and OPERA [119] experiments.

There is no indication in favor of short-baseline $\nu_{\mu}$ and $\bar{\nu}_{\mu}$ disappearance from any experiment. Therefore, the current $\nu_{\mu}$ and $\bar{\nu}_{\mu}$ disappearance data lead to constraints on $\left|U_{\mu 4}\right|^{2}$. We consider the constraints obtained from the CDHSW experiment [120], from the analysis [121] of the data of atmospheric neutrino oscillation experiments, from the analysis of the SciBooNE-MiniBooNE neutrino [122] and antineutrino [123] data, which were included in our previous fits $[12,33,38]$. In addition, we take into account the recent data of the MINOS [35] and IceCube [36] experiments. The MINOS constraint was easily included in our numerical computation by using the ROOT program in the MINOS data release, ${ }^{10}$ which computes the $\chi^{2}$ for input values of the $3+1$ mixing parameters. On the other hand, we had to calculate the IceCube $\chi^{2}$, as described in the following subsection 3.1.

\subsection{Analysis of IceCube data}

The IceCube detector measures the incoming (anti)muons generated by the interaction of atmospheric muon (anti)neutrinos with the surrounding earth and ice, as a function of the neutrino energy and of the zenith angle. For high-energy, up-going atmospheric neutrinos that reach the detector after having crossed the Earth, the ratio $L / E$ is of the same order of that in SBL experiments. In this case, the oscillations arising from the usual atmospheric and solar squared mass differences have a very long wavelength and can be neglected, but the SBL squared mass difference $\Delta m_{41}^{2}$ plays an active role. The sterile neutrino influence on the observed flux is given by the matter effects that modify the oscillation patterns inside the Earth. This happens because the matter potential is different for the different active neutrino flavors, for which the charged and neutral current interactions are not the same [124], and there is no potential for the sterile neutrinos.

We use the 20,145 released IceCube events in the approximate energy range between $320 \mathrm{GeV}$ and $20 \mathrm{TeV}$, detected over 343.7 days in the 86-strings configuration [125] for constraining the active-sterile mixing parameters. The $99.9 \%$ of the IceCube events is expected to come from neutrino-induced muon events, where the neutrinos originate from the decays of atmospheric pions and kaons. The contribution from charmed meson decays is negligible $[125,126]$.

The calculation of the $\chi^{2}$ contribution from IceCube is divided into three parts: the calculation of the theoretical flux for each set of mixing parameters, for which one needs to propagate the atmospheric neutrinos through the Earth, the estimate of the expected number of events in the detector, for which we use the IceCube Monte Carlo data, and finally the computation of the $\chi^{2}$, obtained comparing theoretical and observed events. For all these parts we use the data ${ }^{11}$ and we follow the prescriptions presented in ref. [36].

To obtain the predicted neutrino flux at the detector, we use the $\nu$-SQuIDS code, ${ }^{12}$ a C ++ package based on the Simple Quantum Integro-Differential Solver (SQuIDS) ${ }^{13}$ [127],

\footnotetext{
${ }^{10}$ http://www-numi.fnal.gov/PublicInfo/forscientists.html.

${ }^{11} \mathrm{http}: / /$ icecube.wisc.edu/science/data/IC86-sterile-neutrino/.

${ }^{12}$ http://github.com/Arguelles/nuSQuIDS.

${ }^{13} \mathrm{http}: / /$ github.com/jsalvado/SQuIDS.
} 
that contains all the necessary tools to numerically solve the master equation that rules the neutrino evolution in the Earth [124].

The initial flux we consider is the unoscillated HKKM flux [128-131] with the H3a knee correction [132], that we use for obtaining the initial spectrum of neutrinos from pion and kaon decays. This model is usually referred to as the "Honda-Gaisser" model. We do not employ here the other six atmospheric flux variants that are considered in ref. [36], but we tested that our results do not change significantly if another model is used instead of the Honda-Gaisser one. Since our analysis is based not only on the IceCube data, our final result would be almost unaffected.

The unoscillated flux is propagated inside the Earth with the $\nu$-SQuIDS code, which uses the Preliminary Reference Earth Model [133] for the inner density profile of the Earth. For the neutrino-matter interactions, the charged current cross section is dominated by deep inelastic scattering, which involves neutrino-nucleon scattering. The main uncertainty in this case is in the parton distribution functions. In the $\nu$-SQuIDS code, the perturbative QCD calculation in refs. [134] are used for the neutrino-nucleon cross-section calculation. We do not treat the uncertainties on the Earth density profile and on the deep inelastic scattering cross section.

The full expression for the (anti)neutrino flux at the detector is given by [36] (see also refs. $[135,136])$

$$
\phi_{\text {atm }}^{\nu(\bar{\nu})}\left(E_{\nu(\bar{\nu})}, \cos \theta\right)=N_{0}^{\nu(\bar{\nu})} \mathcal{F}(\delta)\left(\phi_{K}^{\nu(\bar{\nu})}+R_{\pi / K} \phi_{\pi}^{\nu(\bar{\nu})}\right)\left(\frac{E_{\nu(\bar{\nu})}}{E_{m}}\right)^{-\Delta \gamma} .
$$

Here, $\theta$ is the zenith angle and $E_{\nu(\bar{\nu})}$ the energy of the incoming (anti)neutrino, while $\phi_{\pi(K)}^{\nu(\bar{\nu})}$ is the oscillated (anti)neutrino flux from pion (kaon) decays. The free parameters in the above equation are: the neutrino and antineutrino flux normalizations, $N_{0}^{\nu}$ and $N_{0}^{\bar{\nu}}$; the pion-to-kaon ratio, $R_{\pi / K}$; the spectral index correction, $\Delta \gamma$. These are treated as continuous nuisance parameters in our analysis, as explained in ref. [36]. The pivot energy $E_{m}$ is fixed to be approximately near the median of the energy distribution of the measured events, being $E_{m}=2 \mathrm{TeV}$.

The function $\mathcal{F}(\delta)$ parameterizes the atmospheric density uncertainties. This function is assumed to be linear and it is obtained by imposing the AIRS constraints on the atmospheric temperature. ${ }^{14}$ The expression reads as [135]:

$$
\mathcal{F}(\delta)=1+\left(\cos \theta+\cos \theta_{0}\right) \delta\left[1+\frac{E_{\nu(\bar{\nu})}-E_{0}}{E_{1}} \cdot \frac{1}{1+\exp \left(-\kappa\left(\cos \theta+\cos \theta_{0}\right)\right)}\right],
$$

where $E_{0}=360 \mathrm{GeV}, E_{1}=11.279 \mathrm{TeV}, \kappa=200$ and $\cos \theta_{0}=0.4$. The parameter $\delta$ represents the last one of our nuisance parameters.

The theoretical flux is converted into a number of expected events using the Monte Carlo (MC) data released by the IceCube collaboration [36]. The MC data are needed to model the detector capabilities to measure the incoming events as a function of the real

\footnotetext{
${ }^{14}$ https://climatedataguide.ucar.edu/climate-data/airs-and-amsu-tropospheric-air-temperature-andspecific-humidity
} 


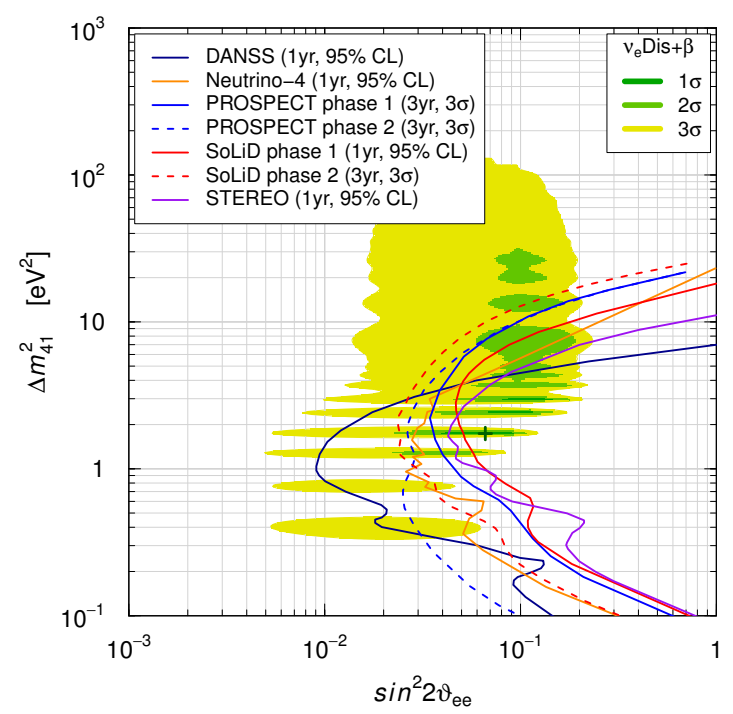

(a)

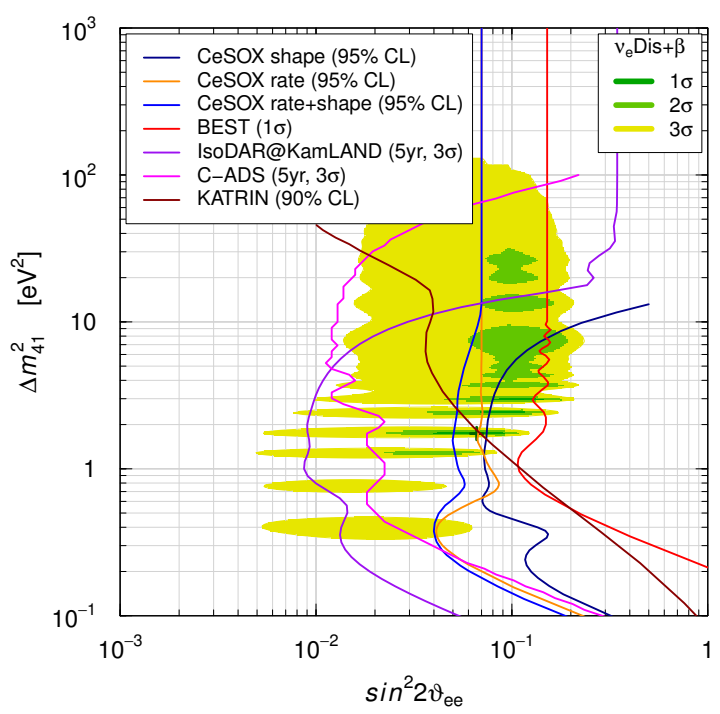

(b)

Figure 6. Sensitivities of future reactor (a) and source (b) experiments compared with the allowed regions in the $\sin ^{2} 2 \vartheta_{e e}-\Delta m_{41}^{2}$ plane in figure $5(\mathrm{~b})$.

energy and zenith angle of the muon (anti)neutrino, and of the corresponding quantities for the reconstructed (anti)muon event. For each combination of mixing and nuisance parameters, we use the MC data to convert the obtained theoretical flux into the expected number of events that we compare with the data as explained below. Since IceCube cannot distinguish a muon from an antimuon, neutrinos and antineutrinos events are summed up together. It is however important to treat properly both the components, since the matter oscillation patterns for neutrinos and antineutrinos are different, with the consequence that the disappearance of neutrinos and antineutrinos is not the same.

We build our $\chi^{2}$ using a binned Poissonian likelihood, written as

$$
\chi^{2}=-2 \ln \mathcal{L}(\boldsymbol{\theta})=2 \sum_{i=1}\left[\mu_{i}(\boldsymbol{\theta})-n_{i}+n_{i} \ln \frac{n_{i}}{\mu_{i}(\boldsymbol{\theta})}\right]
$$

where $n_{i}$ represents the number of observed events in the bin $i$ and $\mu_{i}(\boldsymbol{\theta})$ the corresponding number of expected events as a function of the model parameters $\boldsymbol{\theta}$, that includes both mixing and nuisance parameters. Following ref. [36], we consider a grid with 10 logarithmic bins in the reconstructed energy, with $400 \mathrm{GeV} \leq E_{\mu(\bar{\mu})}^{\mathrm{reco}} \leq 20 \mathrm{TeV}$, and 21 linear bins for the cosine of the reconstructed zenith angle, in the range $-1 \leq \cos \theta_{\mu(\bar{\mu})}^{\text {reco }} \leq 0.2$.

For each combination of the mixing parameters, we minimize the $\chi^{2}$ over the five nuisance parameters described above $\left(N_{0}^{\nu}, N_{0}^{\bar{\nu}}, R_{\pi / K}, \Delta \gamma, \delta\right)$. We adopt a standard NelderMead algorithm for the minimization [137]. It is important to note that for each point in the mixing parameter space we needed to minimize independently over the nuisance parameters. We checked that the preferred values of the nuisance parameters do not vary significantly outside the adopted Gaussian priors [36]. 


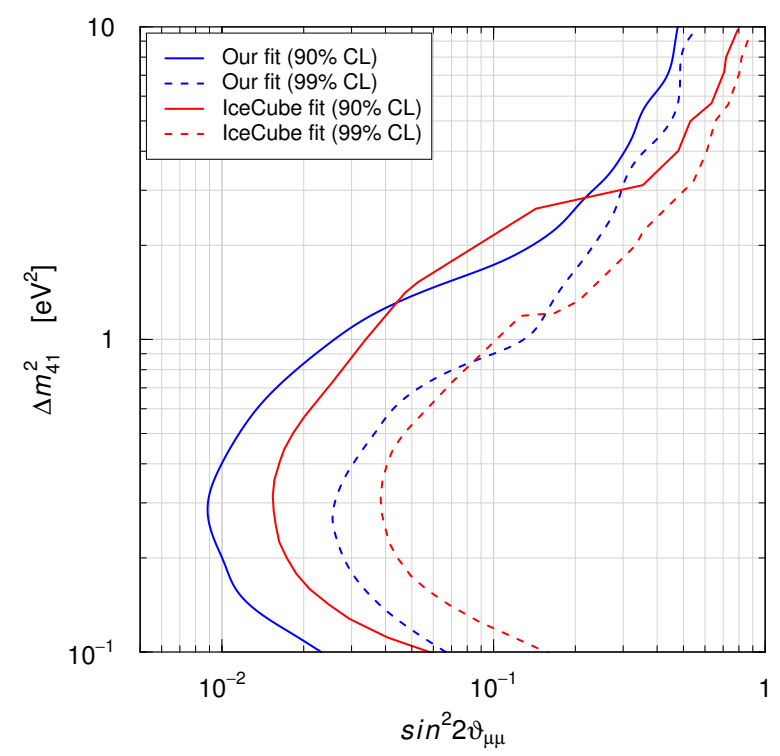

Figure 7. Comparison of the official IceCube $90 \%$ and $99 \%$ CL exclusion curves in the $\sin ^{2} 2 \vartheta_{\mu \mu^{-}}$ $\Delta m_{41}^{2}$ plane [36] with our results. All curves have been obtained assuming $\left|U_{e 4}\right|^{2}=\left|U_{\tau 4}\right|^{2}=0$.

We show in figure 7 the comparison of the official IceCube $90 \%$ and $99 \%$ CL exclusion curves in the $\sin ^{2} 2 \vartheta_{\mu \mu}-\Delta m_{41}^{2}$ plane for $\left|U_{e 4}\right|^{2}=\left|U_{\tau 4}\right|^{2}=0$ [36] with our results. In our analysis of IceCube data we do not vary the efficiency of the Digital Optical Modules because we do not have sufficient information. Despite this fact, one can see from figure 7 that the results of our analysis are in good agreement with those of the IceCube collaboration. Moreover, we emphasize that the IceCube data are just one of the datasets in our global analyses, and small differences in the IceCube analysis do not play a significant role when computing the global fit.

Since the calculation of the $\chi^{2}$ given a set of mixing parameters is a highly demanding computational task, it is impossible to directly include the code that calculates the $\chi^{2}$ of the IceCube data in our complete fitting routine without slowing it down in an unacceptable way. Therefore, we adopted the following method. Since we are more interested in scanning the region near the expected $3+1$ mixing best-fit, we employed the results of the $3+1$ fit of SBL data without IceCube in order to generate with a Markov Chain Monte Carlo (MCMC) 3,000 random points whose distribution covers an area of the parameter space around the expected best-fit region. In order to cover the rest of the full four-dimensional parameter space $\left(\Delta m_{41}^{2} ;\left|U_{e 4}\right|^{2},\left|U_{\mu 4}\right|^{2},\left|U_{\tau 4}\right|^{2}\right)$, we generated uniformly 21,000 more random points. We end up with a set $\mathrm{P}$ of 24,000 points for which we computed the IceCube $\chi^{2}$ in an affordable time. In the complete fitting routine, we computed the IceCube contribution to the $\chi^{2}$ in each point in the full four-dimensional parameter space with a linear interpolation of the $\chi^{2}$ 's of the nearest points in the set $\mathrm{P}$ obtained with a Delaunay triangulation. 


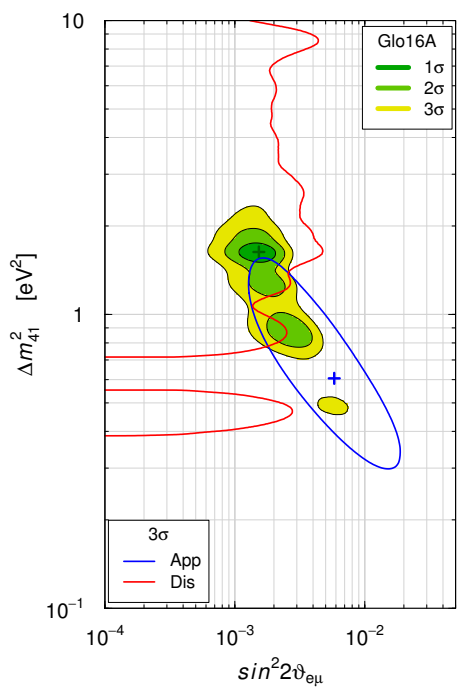

(a)

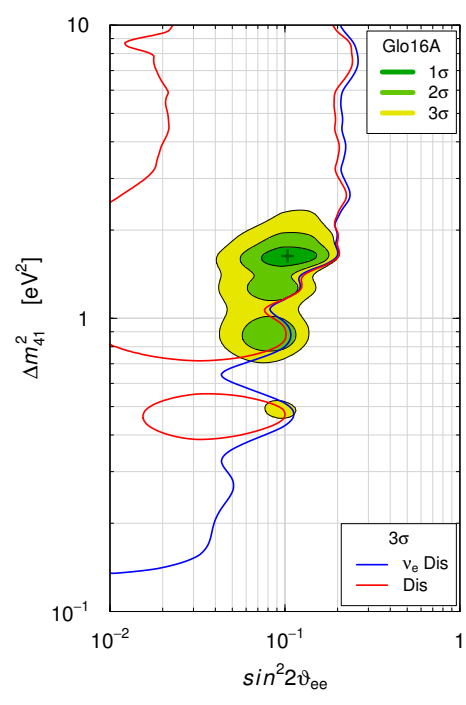

(b)

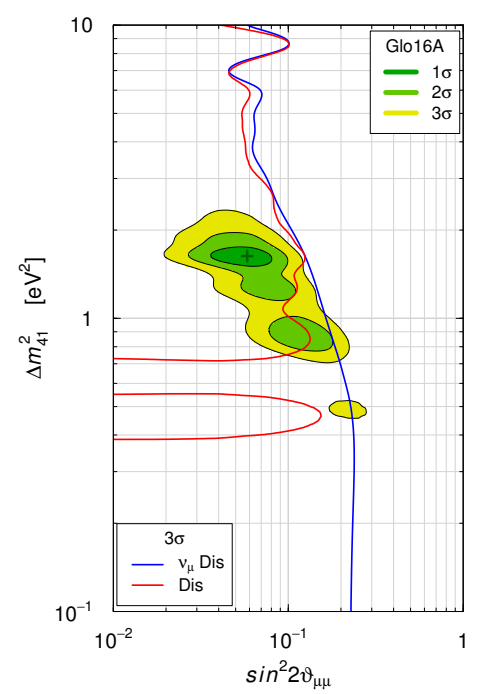

(c)

Figure 8. Allowed regions in the $\sin ^{2} 2 \vartheta_{e \mu}-\Delta m_{41}^{2}$ (a), $\sin ^{2} 2 \vartheta_{e e}-\Delta m_{41}^{2}$ (b), and $\sin ^{2} 2 \vartheta_{\mu \mu}-\Delta m_{41}^{2}$ (c) planes obtained in the $3+1$ global fit "Glo16A" of the 2016 SBL data without the MINOS [35] and IceCube [36] data. There is a comparison with the $3 \sigma$ allowed regions obtained from $\stackrel{(-)}{\nu_{\mu}} \rightarrow \stackrel{(-)}{\nu_{e}}$ SBL appearance data (App) and the $3 \sigma$ constraints obtained from $\stackrel{(-)}{\nu})_{e}$ SBL disappearance data $\left(\nu_{e}\right.$ Dis), $\stackrel{(-)}{\nu}$ SBL disappearance data $\left(\nu_{\mu}\right.$ Dis $)$ and the combined $\stackrel{(-)}{\nu}$ and $\stackrel{(-)}{\nu}$ SBL disappearance data (Dis). The best-fit points of the Glo16A and App fits are indicated by crosses.

\subsection{Fit of the 2016 data set without MINOS and IceCube}

In this subsection we present the results of the $3+1$ global fit "Glo16A" of the appearance and disappearance SBL data available in 2016, ${ }^{15}$ except MINOS [35] and IceCube [36], which will be added in subsection 3.3 in order to clarify their effects on the results of the analysis. In the Glo16A fit we also do not take into account the NEOS [37] data which have been available to us in the beginning of 2017 and will be considered in subsection 3.4.

The results of the Glo16A fit are shown by the first column of table 5 , by figure 8 , and by the solid purple curves in figure 9 , which gives the marginal $\Delta \chi^{2}$ as a function of the mixing parameters $\Delta m_{41}^{2},\left|U_{e 4}\right|^{2}$, and $\left|U_{\mu 4}\right|^{2}$, from which one can obtain the corresponding marginal allowed intervals at different confidence levels.

The global goodness of fit of $4.8 \%$ is acceptable, but there is a relevant appearancedisappearance tension quantified by a parameter goodness of fit of $0.13 \%$. If one is willing to accept such appearance-disappearance tension, one can adopt the allowed regions of the oscillation parameters shown in figure 8 .

The Glo16A fit is an update of the GLO fit presented in ref. [12], with a similar set of data. It can also be compared with the global fit in ref. [40], where a similar set of data was

\footnotetext{
${ }^{15}$ We consider all the $\nu_{e}$ and $\bar{\nu}_{e}$ disappearance data discussed in section 2 , with the exceptions of the T2K near detector constraints [94] on $\sin ^{2} 2 \vartheta_{e e}$, which unfortunately cannot be included in the global fit because they have been obtained under the assumption $\left|U_{\mu 4}\right|^{2}=0$, and of the Mainz [83] and Troitsk [84, 85] $\beta$-decay constraints, which are not needed because the value of $\Delta m_{41}^{2}$ is constrained within a few $\mathrm{eV}^{2}$ by the combination of appearance and disappearance data.
} 


\begin{tabular}{|c|c|c|c|c|}
\hline & Glo16A & Glo16B & Glo17 & PrGlo17 \\
\hline$\chi_{\min }^{2}$ & 288.4 & 556.9 & 622.1 & 595.1 \\
\hline $\mathrm{NDF}$ & 250 & 525 & 585 & 579 \\
\hline GoF & $4.8 \%$ & $16 \%$ & $14 \%$ & $31 \%$ \\
\hline$\Delta m_{41}^{2}$ & 1.6 & 1.6 & 1.7 & 1.7 \\
\hline$\left|U_{e 4}\right|^{2}$ & 0.027 & 0.028 & 0.021 & 0.020 \\
\hline$\left|U_{\mu 4}\right|^{2}$ & 0.015 & 0.014 & 0.016 & 0.015 \\
\hline $\sin ^{2} 2 \vartheta_{e \mu}$ & 0.0015 & 0.0015 & 0.0013 & 0.0012 \\
\hline $\sin ^{2} 2 \vartheta_{e e}$ & 0.10 & 0.11 & 0.080 & 0.079 \\
\hline $\sin ^{2} 2 \vartheta_{\mu \mu}$ & 0.058 & 0.054 & 0.062 & 0.058 \\
\hline$\Delta \chi_{\mathrm{NO}}^{2}$ & 53.1 & 51.9 & 51.7 & 47.4 \\
\hline $\mathrm{NDF}_{\mathrm{NO}}$ & 3 & 4 & 4 & 4 \\
\hline$n \sigma_{\mathrm{NO}}$ & 6.7 & 6.4 & 6.4 & 6.1 \\
\hline$\left(\chi_{\min }^{2}\right)_{\mathrm{App}}$ & 94.3 & 94.3 & 94.3 & 77.3 \\
\hline $\mathrm{NDF}_{\text {App }}$ & 84 & 84 & 84 & 78 \\
\hline $\mathrm{GoF}_{\mathrm{App}}$ & $21 \%$ & $21 \%$ & $21 \%$ & $50 \%$ \\
\hline$\Delta m_{41}^{2}$ & 0.61 & 0.61 & 0.61 & 0.97 \\
\hline $\sin ^{2} 2 \vartheta_{e \mu}$ & 0.0058 & 0.0058 & 0.0058 & 0.0026 \\
\hline$\left(\chi_{\min }^{2}\right)_{\text {Dis }}$ & 180.8 & 448.3 & 510.6 & 510.6 \\
\hline $\mathrm{NDF}_{\text {Dis }}$ & 163 & 439 & 499 & 499 \\
\hline $\mathrm{GoF}_{\text {Dis }}$ & $16 \%$ & $37 \%$ & $35 \%$ & $35 \%$ \\
\hline$\Delta m_{41}^{2}$ & 1.7 & 1.7 & 1.7 & 1.7 \\
\hline$\left|U_{e 4}\right|^{2}$ & 0.025 & 0.025 & 0.017 & 0.017 \\
\hline$\left|U_{\mu 4}\right|^{2}$ & 0.011 & 0.0088 & 0.0073 & 0.0073 \\
\hline $\sin ^{2} 2 \vartheta_{e \mu}$ & 0.0011 & 0.00086 & 0.00048 & 0.00048 \\
\hline $\sin ^{2} 2 \vartheta_{e e}$ & 0.097 & 0.097 & 0.065 & 0.065 \\
\hline $\sin ^{2} 2 \vartheta_{\mu \mu}$ & 0.042 & 0.035 & 0.029 & 0.029 \\
\hline$\Delta \chi_{\mathrm{PG}}^{2}$ & 13.4 & 14.4 & 17.2 & 7.2 \\
\hline $\mathrm{NDF}_{\mathrm{PG}}$ & 2 & 2 & 2 & 2 \\
\hline $\mathrm{GoF}_{\mathrm{PG}}$ & $0.13 \%$ & $0.075 \%$ & $0.019 \%$ & $2.7 \%$ \\
\hline
\end{tabular}

Table 5. Results of the $3+1$ global Glo16A, Glo16B, Glo17, and PrGlo17 fits of SBL data discussed, respectively, in subsections $3.2,3.3,3.4$, and 3.5. The first group of rows gives: the minimum $\chi^{2}$ $\left(\chi_{\min }^{2}\right)$, the number of degrees of freedom (NDF), the goodness of fit $(\mathrm{GoF})$, the best fit values of the mixing parameters $\Delta m_{41}^{2},\left|U_{e 4}\right|^{2},\left|U_{\mu 4}\right|^{2}$, and of the oscillation amplitudes $\sin ^{2} 2 \vartheta_{e \mu}, \sin ^{2} 2 \vartheta_{e e}$, $\sin ^{2} 2 \vartheta_{\mu \mu}$. The second group of rows gives the $\chi^{2}$ difference $\Delta \chi_{\mathrm{NO}}^{2}$ between the $\chi^{2}$ of no oscillations and $\chi_{\min }^{2}$ and the resulting number of $\sigma$ 's $\left(n \sigma_{\mathrm{NO}}\right)$ for $\mathrm{NDF}_{\mathrm{NO}}$ degrees of freedom corresponding to the number of fitted parameters. The third and fourth group of rows give, respectively, the results of different $3+1$ fits of appearance (App) and disappearance (Dis) data. The fifth group of rows gives the results for the appearance-disappearance parameter goodness of fit [178]: the $\chi^{2}$ difference $\Delta \chi_{\mathrm{PG}}^{2}$ and the resulting goodness of fit $\mathrm{GoF}_{\mathrm{PG}}$ for $\mathrm{NDF}_{\mathrm{PG}}$ degrees of freedom. 


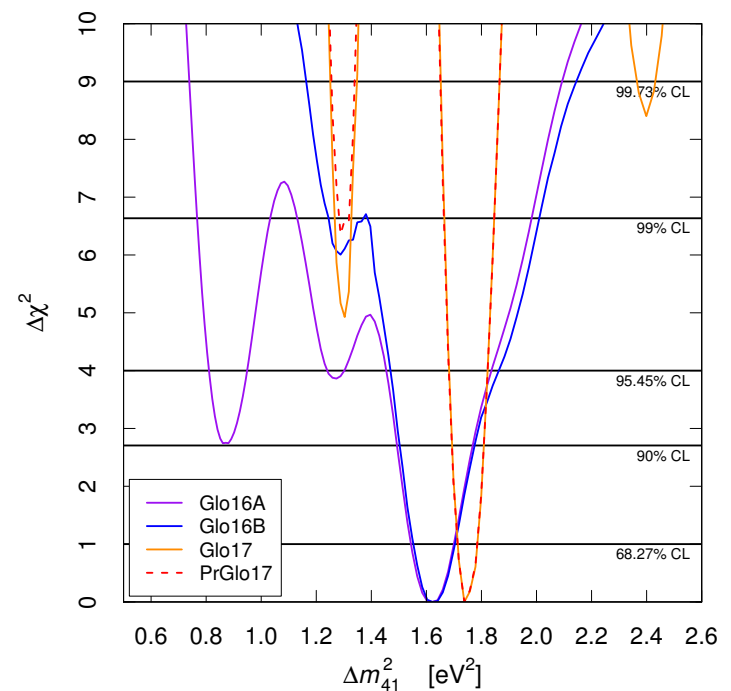

(a)

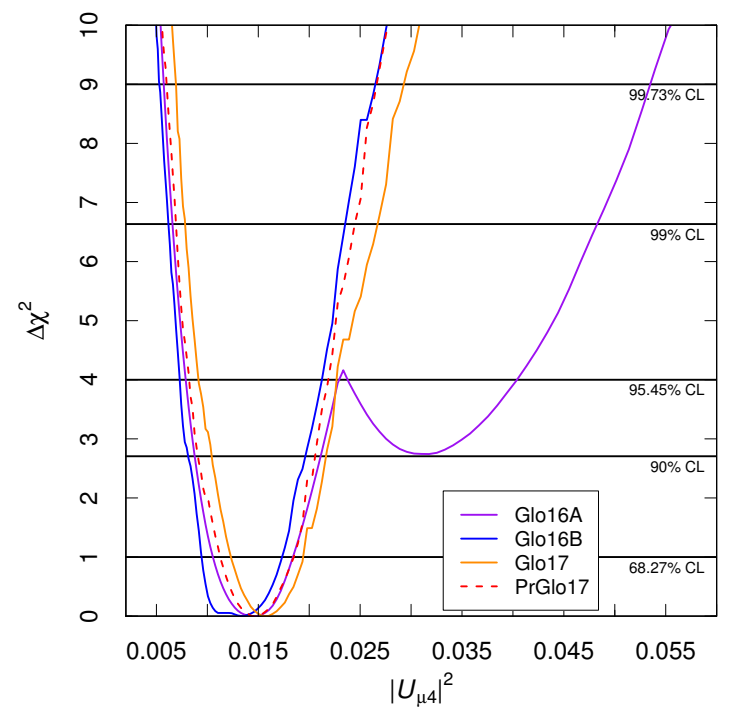

(c)

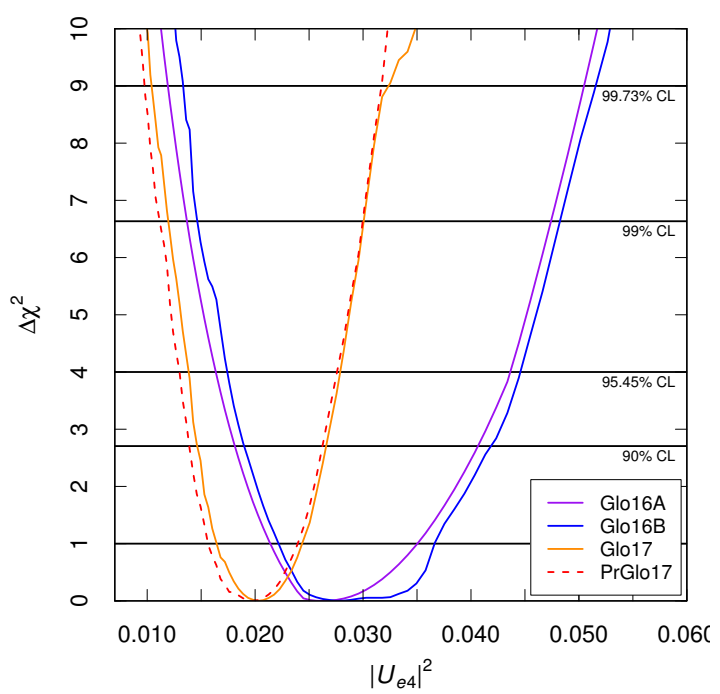

(b)

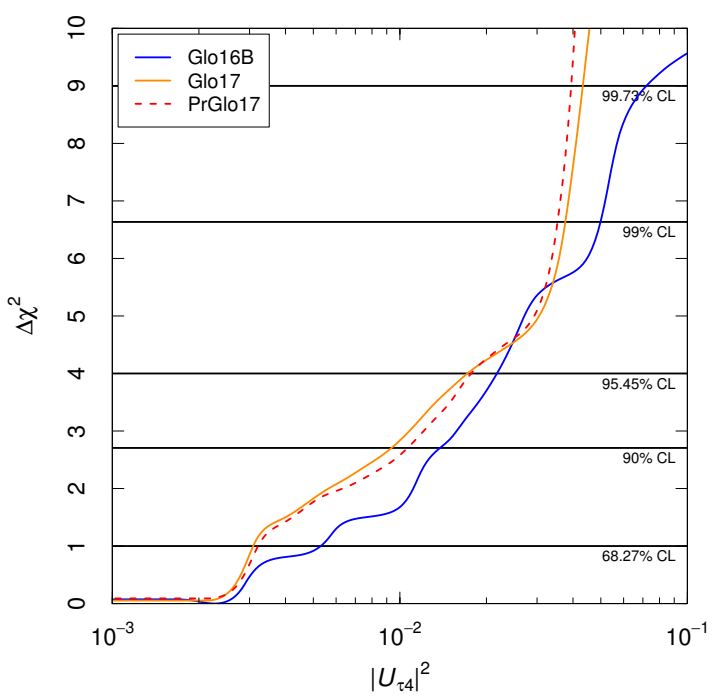

(d)

Figure 9. Marginal $\Delta \chi^{2}=\chi^{2}-\chi_{\min }^{2}$ as a function of the mixing parameters $\Delta m_{41}^{2}$ (a), $\left|U_{e 4}\right|^{2}$ (b), $\left|U_{\mu 4}\right|^{2}$ (c), and $\left|U_{\tau 4}\right|^{2}$ (d). The black horizontal lines show the $\Delta \chi^{2}$ for one degree of freedom corresponding to the indicated confidence level (CL).

considered. With respect to ref. [40], we find larger allowed regions for $\Delta m^{2} \lesssim 2 \mathrm{eV}^{2}$ and we do not have the allowed region at $\Delta m^{2} \approx 6 \mathrm{eV}^{2}$ found in ref. [40] at $99 \%$ CL. However, there is an approximate agreement of our results with those of ref. [40], with a remarkable closeness of the best-fit point in the mixing parameter space.

\subsection{Effects of MINOS and IceCube}

In this subsection we present the $3+1$ global fit "Glo16B" with the addition of the 2016 data of the MINOS [35] and IceCube [36] experiments. The results are shown by the second column of table 5 , by figure 10, and by the solid blue curves in figure 9 . 


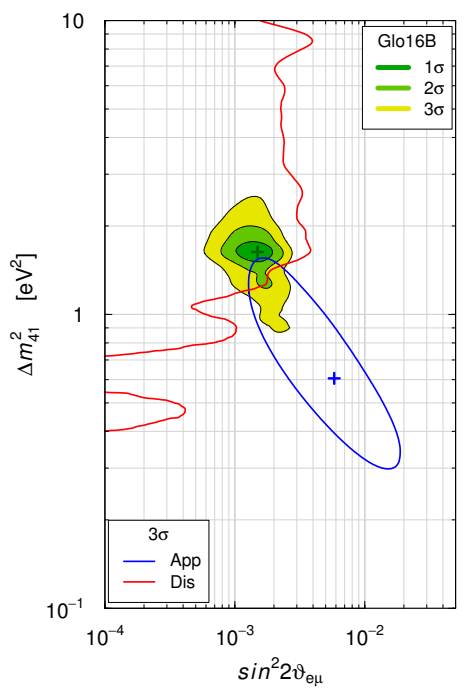

(a)

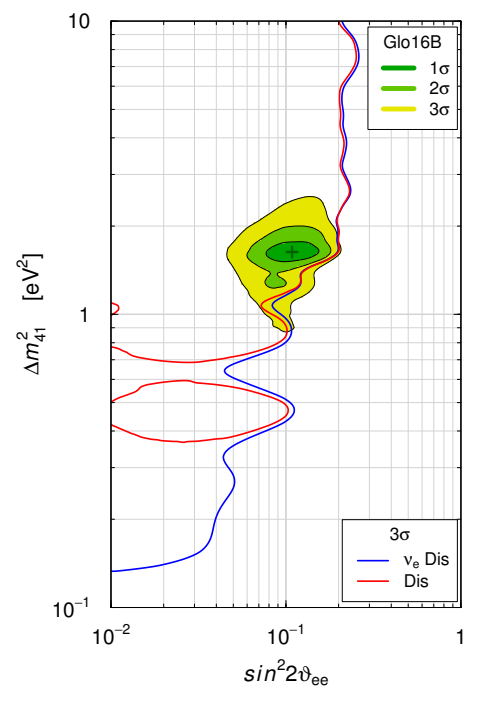

(b)

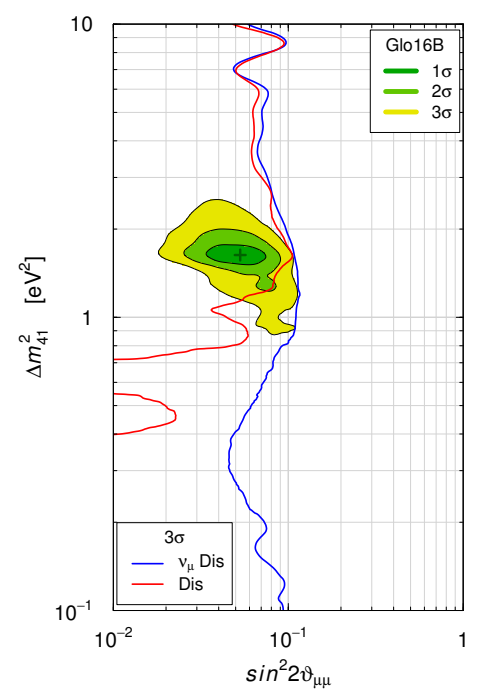

(c)

Figure 10. Allowed regions in the $\sin ^{2} 2 \vartheta_{e \mu}-\Delta m_{41}^{2}$ (a), $\sin ^{2} 2 \vartheta_{e e^{-}} \Delta m_{41}^{2}$ (b), and $\sin ^{2} 2 \vartheta_{\mu \mu}-\Delta m_{41}^{2}$ (c), planes obtained in the $3+1$ global fit "Glo16B" of all 2016 SBL data. There is a comparison with the $3 \sigma$ allowed regions obtained from $\stackrel{(-)}{\nu}) \rightarrow(-))$ SBL appearance data (App) and the $3 \sigma$ constraints obtained from $\stackrel{(-)}{\nu}$ SBL disappearance data $\left(\nu_{e}\right.$ Dis $), \stackrel{(-)}{\nu}$ S SBL disappearance data $\left(\nu_{\mu}\right.$ Dis $)$ and the combined $\stackrel{(-)}{\nu_{e}}$ and $\stackrel{(-)}{\nu}_{\mu}$ SBL disappearance data (Dis). The best-fit points of the Glo16B and App fits are indicated by crosses.

Comparing figure 10(c) with figure 8(c), one can see that the addition of the MINOS and IceCube data leads to the exclusion of the low- $\Delta m_{41}^{2}-$ high- $\sin ^{2} 2 \vartheta_{\mu \mu}$ part of the allowed region, as expected (see the discussion in section 1). On the other hand, the high- $\Delta m_{41}^{2}-$ low- $\sin ^{2} 2 \vartheta_{\mu \mu}$ part of the allowed region is practically unaffected by the MINOS and IceCube constraints. As a consequence, also the low- $\Delta m_{41}^{2}-$ high- $\sin ^{2} 2 \vartheta_{e \mu}$ part of the allowed region in figure 8(a) is excluded in figure 10(a), whereas the high- $\Delta m_{41}^{2}-$ low- $\sin ^{2} 2 \vartheta_{e \mu}$ part of the allowed region is practically unaffected.

From table 5 one can see that including the MINOS and IceCube data increases the appearance-disappearance tension by lowering the parameter goodness of fit from $0.13 \%$ to $0.075 \%$. This is a consequence of the reduction of the upper limit of the allowed range of $\left|U_{\mu 4}\right|^{2}$ in the Glo16B fit with respect to the Glo16A fit shown in figure 9(c).

Figure 11(a) shows the effect of adding to the data set of the Glo16A fit the MINOS and IceCube data separately and together. One can see that the IceCube data are slightly more effective than the MINOS data in reducing the low- $\Delta m_{41}^{2}-$ high- $\sin ^{2} 2 \vartheta_{e \mu}$ part of the allowed region.

The MINOS and IceCube data give information not only on the $3+1$ mixing parameters $\Delta m_{41}^{2},\left|U_{e 4}\right|^{2}$, and $\left|U_{\mu 4}\right|^{2}$ that we have considered so far, but also on $\left|U_{\tau 4}\right|^{2}$. The sensitivity to $\left|U_{\tau 4}\right|^{2}$ is due in MINOS to the neutral-current event sample [35] and in IceCube to the matter effects for high-energy neutrinos propagating in the Earth, which depend on all the elements of the mixing matrix [138-145]. Limits on the value of $\left|U_{\tau 4}\right|^{2}$ have been obtained in the analyses of the atmospheric neutrino data of the Super-Kamiokande [146] and IceCube 


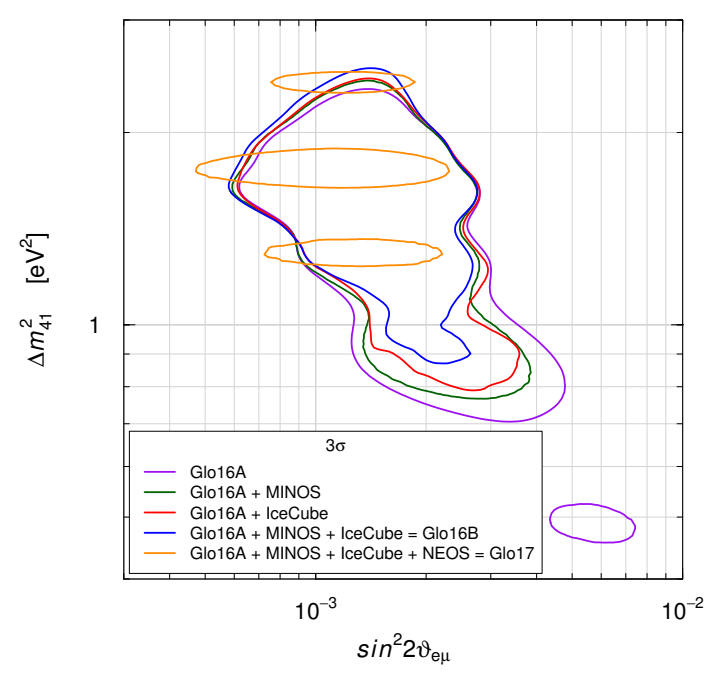

(a)

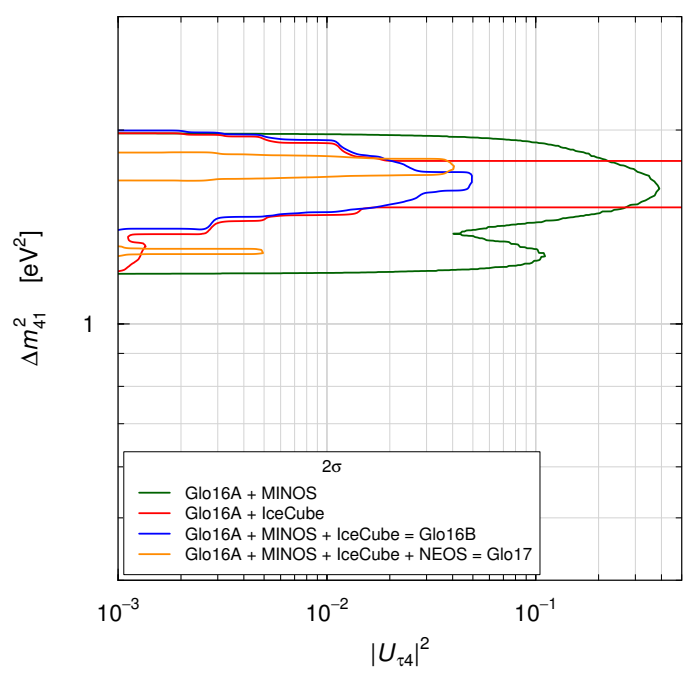

(b)

Figure 11. Comparison of (a) the $3 \sigma$ allowed regions in the $\sin ^{2} 2 \vartheta_{e \mu}-\Delta m_{41}^{2}$ plane and (b) the $2 \sigma$ allowed regions in the $\left|U_{\tau 4}\right|^{2}-\Delta m_{41}^{2}$ plane obtained by adding to the data set of the Glo16A fit the MINOS and IceCube data separately and together, and by adding also the NEOS data.

DeepCore [147] experiments, in the analysis of the data of the MINOS experiment [35, 148, 149], and in the phenomenological fits in refs. [39, 49]. There is also a bound on $\sin ^{2} 2 \vartheta_{\mu \tau}=4\left|U_{\mu 4}\right|^{2}\left|U_{\tau 4}\right|^{2}$ given by the absence of a $3+1$ excess of $\nu_{\mu} \rightarrow \nu_{\tau}$ oscillations in the OPERA experiment [150].

Figure 9(d) shows the marginal $\Delta \chi^{2}$ as a function of $\left|U_{\tau 4}\right|^{2}$ in our Glo16B fit, from which one can see that we obtain the stringent upper bound

$$
\left|U_{\tau 4}\right|^{2} \lesssim 0.022(0.071) \quad \text { at } 2 \sigma(3 \sigma)
$$

At $90 \%$ CL we have $\left|U_{\tau 4}\right|^{2} \lesssim 0.014$ and $\vartheta_{34} \lesssim 7.4^{\circ}$ in the common parameterization of the $4 \times 4$ unitary mixing matrix used in ref. [39]. This bound on $\vartheta_{34}$ is about the same as that reported in ref. [39] for $\Delta m_{41}^{2} \approx 6 \mathrm{eV}^{2}$. However, we do not find a $90 \%$ CL allowed region of the mixing parameters at $\Delta m_{41}^{2} \approx 6 \mathrm{eV}^{2}$ and our bound on $\vartheta_{34}$ applies for any value of $\Delta m_{41}^{2}$.

Figure 11(b) shows the correlated bounds on $\left|U_{\tau 4}\right|^{2}$ and $\Delta m_{41}^{2}$ that we obtain considering the MINOS and IceCube data separately and together. One can see that the IceCube data give more stringent constraints on $\left|U_{\tau 4}\right|^{2}$ than the MINOS data for $\Delta m_{41}^{2} \lesssim 1.5 \mathrm{eV}^{2}$.

\subsection{Effects of NEOS}

We finally consider also the NEOS [37] data and obtain the $3+1$ global fit "Glo17" which includes all data available so far in 2017. The results are shown by the third column of table 5 , by figure 12 , and by the solid orange curves in figure 9 .

Comparing figure 12 with figure 10, it is evident that the inclusion of the NEOS constraints has a dramatic effect on the allowed regions, leading to the fragmentation of the allowed region in three islands with narrow $\Delta m_{41}^{2}$ widths. The best-fit island is at 


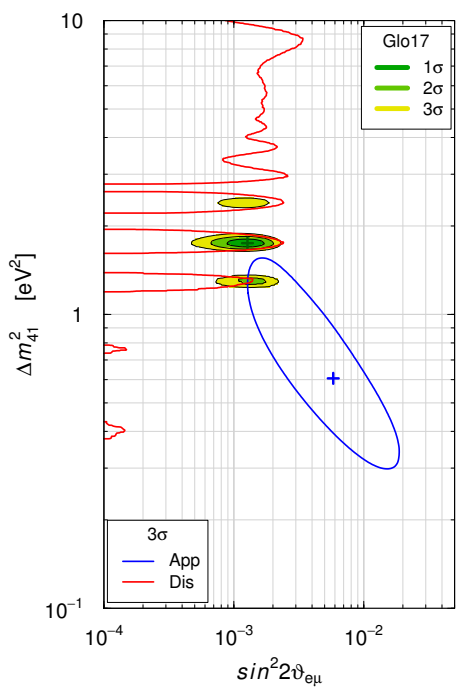

(a)

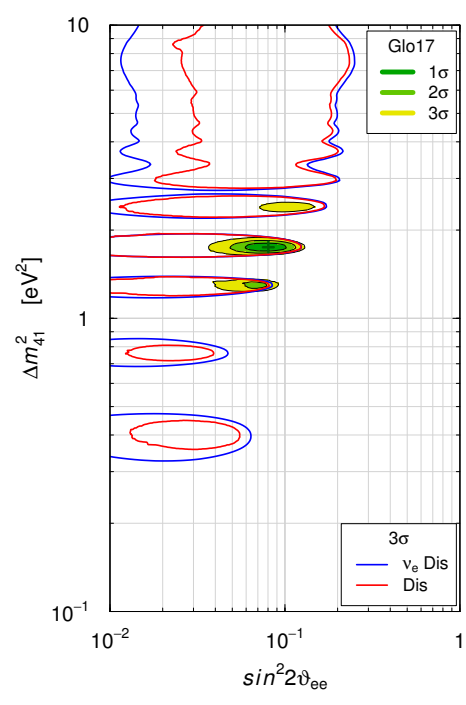

(b)

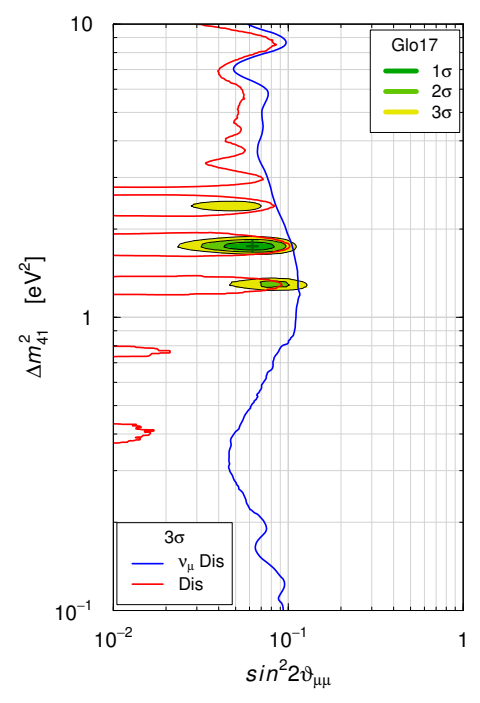

(c)

Figure 12. Allowed regions in the $\sin ^{2} 2 \vartheta_{e \mu}-\Delta m_{41}^{2}$ (a), $\sin ^{2} 2 \vartheta_{e e}-\Delta m_{41}^{2}$ (b), and $\sin ^{2} 2 \vartheta_{\mu \mu}-\Delta m_{41}^{2}$ (c), planes obtained in the $3+1$ global fit "Glo17" of all SBL data. There is a comparison with the $3 \sigma$ allowed regions obtained from $\stackrel{(-)}{\nu} \rightarrow \stackrel{(-)}{\nu}$ SBL appearance data (App) and the $3 \sigma$ constraints obtained from $\stackrel{(-)}{\nu}$ SBL disappearance data $\left(\nu_{e}\right.$ Dis $\left.), \stackrel{(-)}{\nu}\right)$ SBL disappearance data $\left(\nu_{\mu}\right.$ Dis $)$ and the combined $\left(\nu_{e}\right)$ and $\nu_{\mu}$ SBL disappearance data (Dis). The best-fit points of the Glo17 and App fits are indicated by crosses.

$\Delta m_{41}^{2} \approx 1.7 \mathrm{eV}^{2}$. There is an island allowed at $2 \sigma$ at $\Delta m_{41}^{2} \approx 1.3 \mathrm{eV}^{2}$, and an island allowed at $3 \sigma$ at $\Delta m_{41}^{2} \approx 2.4 \mathrm{eV}^{2}$. Moreover, the NEOS constraints shifts the $3 \sigma$ allowed range of $\left|U_{e 4}\right|^{2}$ from $0.014-0.051$ in the Glo16B fit to $0.011-0.032$ in the Glo17 fit, as shown in figure 9 . Therefore, the appearance-disappearance tension is increased, as shown by the $0.019 \%$ parameter goodness of fit in table 5 . Since this low value of the appearancedisappearance parameter goodness of fit is hardly acceptable, we are led to consider, in the next subsection, the "pragmatic approach" proposed in ref. [33].

\subsection{Pragmatic fit}

In this section we consider the "pragmatic approach" [33] in which the low-energy bins of the MiniBooNE experiment [50, 51] which have an anomalous excess of $\stackrel{(-)}{\nu_{e}}$-like events are omitted from the global fit. As shown in figure $1 \mathrm{~b}$ of ref. [38], the region allowed by the appearance data shifts towards larger values of $\Delta m_{41}^{2}$ and smaller values of $\sin ^{2} 2 \vartheta_{e \mu}$ when the MiniBooNE low-energy bins are omitted from the fit. As a result, the overlap of the appearance and disappearance allowed regions increases, relieving the appearancedisappearance tension.

One can question the scientific correctness of the data selection in the pragmatic approach, but we note that the MiniBooNE low-energy excess is widely considered to be suspicious ${ }^{16}$ because of the large background. Some of this background can be due to

\footnotetext{
${ }^{16}$ Part of the MiniBooNE low-energy anomaly may be explained by taking into account nuclear effects in the energy reconstruction [151, 152], but this effect is not sufficient to solve the problem [153].
} 


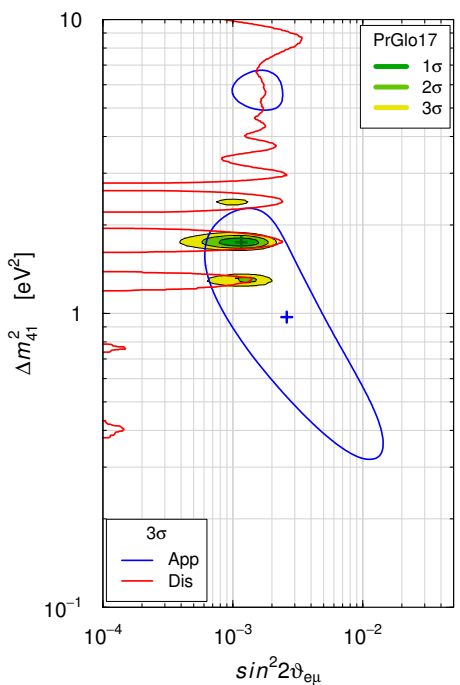

(a)

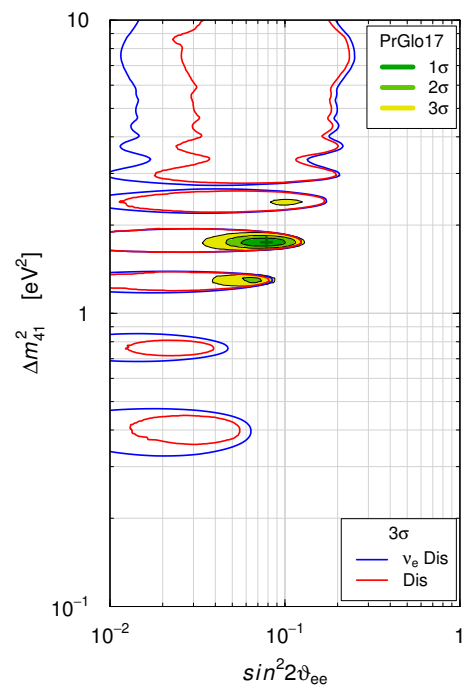

(b)

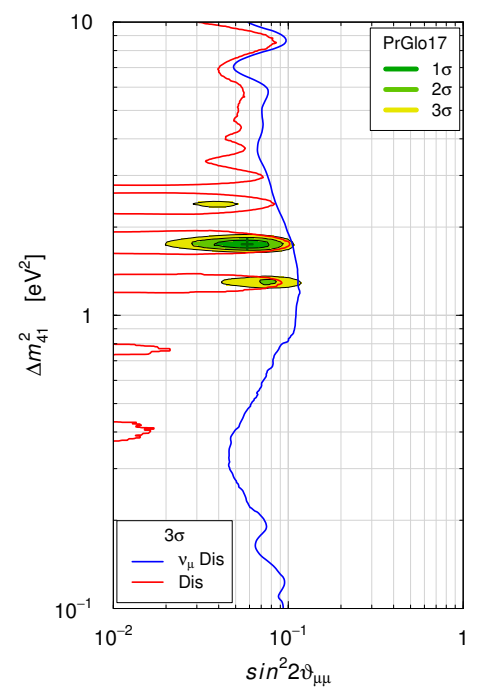

(c)

Figure 13. Allowed regions in the $\sin ^{2} 2 \vartheta_{e \mu}-\Delta m_{41}^{2}$ (a), $\sin ^{2} 2 \vartheta_{e e}-\Delta m_{41}^{2}$ (b), and $\sin ^{2} 2 \vartheta_{\mu \mu}-\Delta m_{41}^{2}$ (c), planes obtained in the pragmatic $3+1$ global fit "PrGlo17" of SBL data. There is a comparison with the $3 \sigma$ allowed regions obtained from $\stackrel{(-)}{\nu}) \rightarrow \stackrel{(-)}{\nu}$ SBL appearance data (App) and the $3 \sigma$ constraints obtained from $\stackrel{(-)}{\nu}$ SBL disappearance data $\left(\nu_{e}\right.$ Dis $\left.), \stackrel{(-)}{\nu}\right)$ SBL disappearance data $\left(\nu_{\mu}\right.$ Dis) and the combined $\stackrel{(-)}{\nu_{e}}$ and $\stackrel{(-)}{\nu}$ SBL disappearance data (Dis). The best-fit points of the PrGlo17 and App fits are indicated by crosses.

photon events which are indistinguishable from $\stackrel{(-)}{\nu_{e}}$ events in the MiniBooNE liquid scintillator detector. These photons can be generated by the decays of $\pi^{0}$ 's produced by the neutral-current interactions of the $\stackrel{(-)}{\nu_{\mu}}$ beam. When only one of the two photons emitted in the $\pi^{0}$ decay is visible, its signal cannot be distinguished from a $\stackrel{(-)}{\nu}$ event in a liquidscintillator detector. The suspicion that this photon background may be responsible for the MiniBooNE low-energy excess motivated the realization of the MicroBooNE experiment at Fermilab [52], which is able to distinguish between photon and $\stackrel{(-)}{\nu}$ events by using a Liquid Argon Time Projection Chamber (LArTPC). Waiting for the results of this experiment, we think that it is reasonable to adopt the pragmatic approach of omitting from the global fit the MiniBooNE low-energy data.

The results of the pragmatic $3+1$ global fit "PrGlo17", which includes the MINOS, IceCube and NEOS data, are shown by the fourth column of table 5 , by figure 13 , and by the dashed red curves in figure 9 .

From table 5 one can see that, as expected, the exclusion from the fit of the MiniBooNE low-energy data leads to an increase of the parameter goodness of fit from the unacceptable $0.019 \%$ of the Glo17 fit to the acceptable $2.7 \%$ of the PrGlo17 fit. There is still a mild appearance-disappearance tension, but the tolerable value of parameter goodness of fit leads us to consider the PrGlo17 fit as acceptable.

Comparing the allowed regions of the oscillation parameters in figure 13 for the PrGlo17 fit with those in figure 12 for the Glo17 fit and the corresponding marginal $\Delta \chi^{2}$ curves in figure 9 , one can see that the differences are small. As a consequence of the larger overlap of 


\begin{tabular}{|cccc|}
\hline $\mathrm{CL}$ & $\left|U_{e 4}\right|^{2}$ & $\left|U_{\mu 4}\right|^{2}$ & $\left|U_{\tau 4}\right|^{2}$ \\
\hline $68.27 \%(1 \sigma)$ & $0.016-0.024$ & $0.011-0.018$ & $\lesssim 0.0032$ \\
$95.45 \%(2 \sigma)$ & $0.013-0.028$ & $0.0083-0.022$ & $\lesssim 0.018$ \\
$99.73 \%(3 \sigma)$ & $0.0098-0.031$ & $0.0060-0.026$ & $\lesssim 0.039$ \\
\hline
\end{tabular}

Table 6. Marginal allowed intervals of the mixing parameters $\left|U_{e 4}\right|^{2},\left|U_{\mu 4}\right|^{2}$, and $\left|U_{\tau 4}\right|^{2}$ obtained in the pragmatic $3+1$ global fit "PrGlo17" of SBL data.

the regions allowed by the fits of appearance and disappearance data, the PrGlo17 fit has a minimum $\chi^{2}$ significantly smaller than the Glo17 fit, which leads to an increased preference of the best-fit island at $\Delta m_{41}^{2} \approx 1.7 \mathrm{eV}^{2}$, to a small shrink of the island at $\Delta m_{41}^{2} \approx 1.3 \mathrm{eV}^{2}$, and at a significant reduction of the island at $\Delta m_{41}^{2} \approx 2.4 \mathrm{eV}^{2}$ (the corresponding $3 \sigma$ interval for one degree of freedom allowed by the marginal $\Delta \chi^{2}$ in figure 9 (a) disappears).

Table 6 gives the marginal allowed intervals of the mixing parameters $\left|U_{e 4}\right|^{2},\left|U_{\mu 4}\right|^{2}$, and $\left|U_{\tau 4}\right|^{2}$. The stringent upper bounds on $\left|U_{\tau 4}\right|^{2}$ slightly improve those found in the Glo16B fit (see eq. (3.4) and figure 9(d)). At 90\% CL we have $\left|U_{\tau 4}\right|^{2} \lesssim 0.011$ and $\vartheta_{34} \lesssim 6^{\circ}$.

We consider the results of the PrGlo17 fit as the current status of our $3+1$ analysis of short-baseline neutrino oscillation data. Figure 14 shows a comparison of the sensitivities of future experiments with the PrGlo17 allowed regions of figure 13 for: $14(\mathrm{a}) \stackrel{(-)}{\nu}{ }_{\mu} \rightarrow \stackrel{(-)}{\nu_{e}}$ transitions (SBN [154], nuPRISM [155], JSNS 2 [156]); 14(b) $\stackrel{(-)}{\nu}$ disappearance (SBN [154], KPipe [157]); 14(c),(d) $\stackrel{(-)}{\nu}$ disappearance (DANSS [98], Neutrino-4 [104], PROSPECT [99], SoLid [105], STEREO [106], CeSOX [102], BEST [103] IsoDAR@KamLAND [107], CADS [108], KATRIN [109]). It is clear that these experiments will give definitive information on the existence of active-sterile short-baseline oscillations connected with the LSND, Gallium and reactor anomalies.

\section{Conclusions}

In this paper we updated the global fit of short-baseline neutrino oscillation data in the framework of $3+1$ active-sterile neutrino mixing [7, 12, 32, 33].

We considered first, in section 2 , the data on $\nu_{e}$ and $\bar{\nu}_{e}$ disappearance which include the Gallium neutrino anomaly data [3-7] and the reactor antineutrino anomaly data [8]. The resulting allowed region in the $\sin ^{2} 2 \vartheta_{e e}-\Delta m_{41}^{2}$ plane is rather wide, as shown in figure 5(a), but it is smaller than that found in our previous analysis [7], mainly as a result of the constraints given by the recent NEOS [37] experiment. The allowed region obtained with neutrino oscillation data alone has no upper bound for $\Delta m_{41}^{2}$, but it can be limited [32] using the constraints found in the Mainz [83] and Troitsk [84, 85] $\beta$-decay experiments, as shown in figure $5(\mathrm{~b})$. We found the upper limit $\Delta m_{41}^{2} \lesssim 148 \mathrm{eV}^{2}$ at $3 \sigma$. Hence, as shown in figure 6 , the ongoing reactor, source and $\beta$-decay experiments can clarify in a definitive way the existence of short-baseline $\stackrel{(-)}{\nu_{e}}$ disappearance due to active-sterile neutrino mixing.

We presented also, in section 3 , the results of global fits of all the available $\stackrel{(-)}{\nu_{\mu}} \rightarrow \stackrel{(-)}{\nu}$ appearance data,,$\stackrel{(-)}{\nu}$ disappearance data, in addition to the $\stackrel{(-)}{\nu_{e}}$ disappearance data considered in section 2. We discussed the effects on the global fits of the recent data of the MINOS [35], 


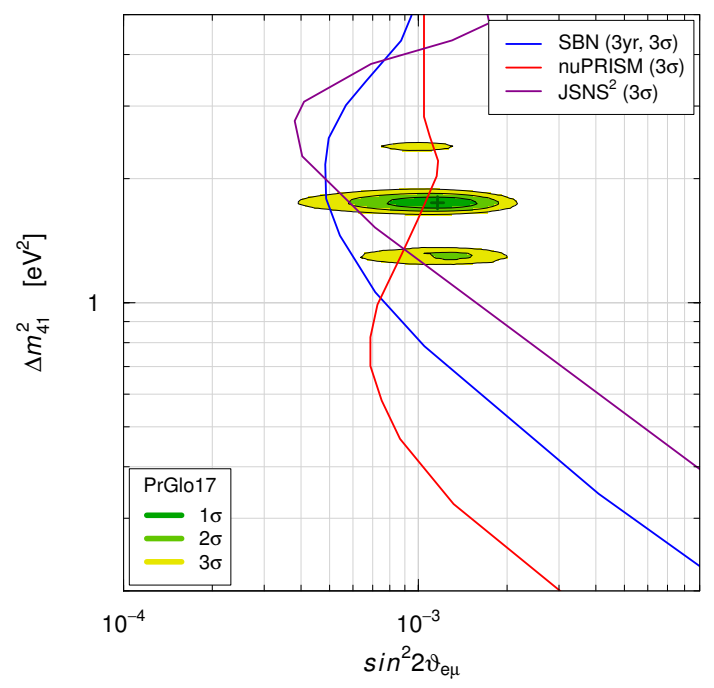

(a)

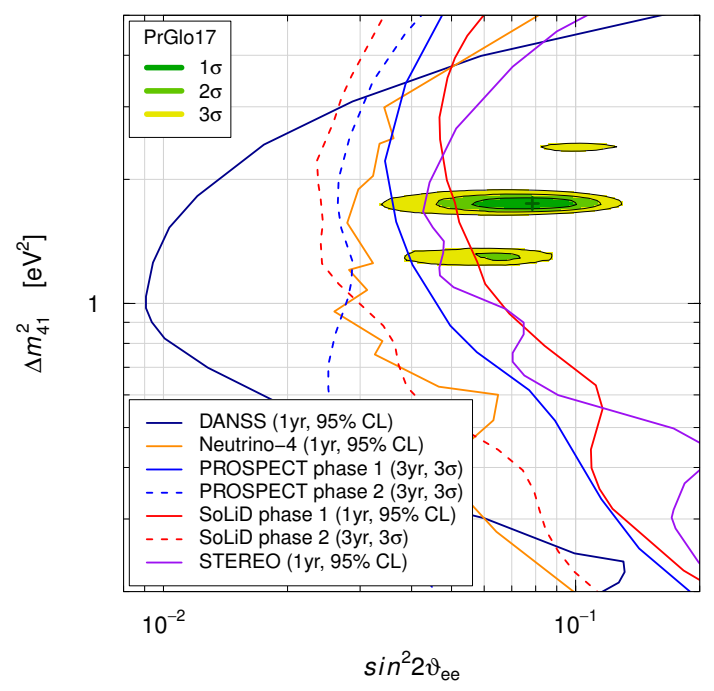

(c)

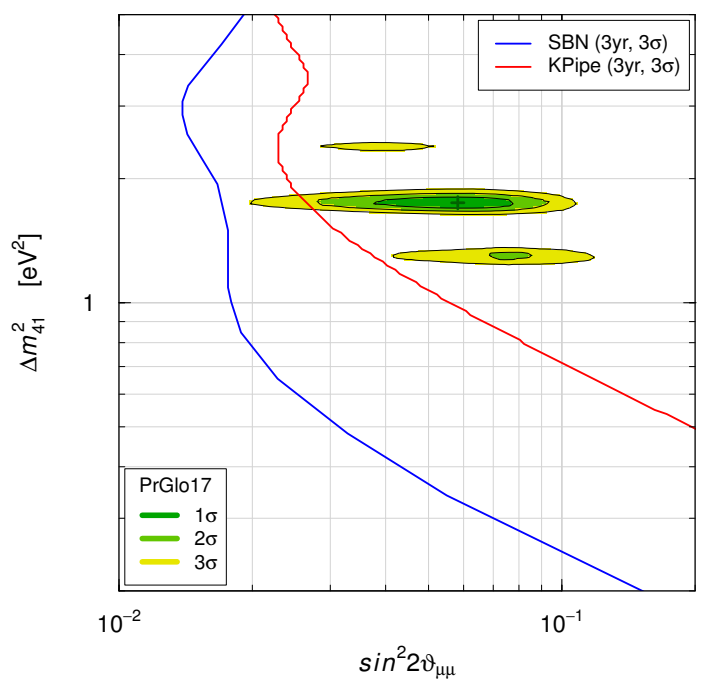

(b)

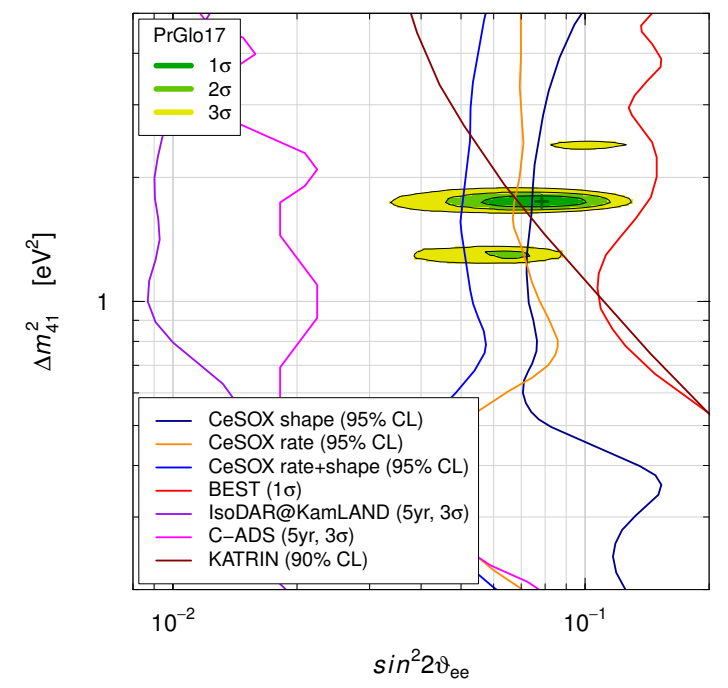

(d)

Figure 14. Sensitivities of future experiments compared with the PrGlo17 allowed regions of figure 13.

IceCube [36], and NEOS [37] experiments. As expected, the MINOS, IceCube and NEOS data aggravate the appearance-disappearance tension, which becomes tolerable only in the pragmatic PrGlo17 fit discussed in subsection 3.5, which is our recommended result.

We found that, as expected [38, 39], the MINOS and IceCube constraints on $\stackrel{(-)}{\nu_{\mu}}$ disappearance disfavor the low- $\Delta m_{41}^{2}-$ high- $\sin ^{2} 2 \vartheta_{\mu \mu}$ and the low- $\Delta m_{41}^{2}-$ high- $\sin ^{2} 2 \vartheta_{e \mu}$ parts of the allowed region. The addition of the NEOS data has the more dramatic effect of reducing the allowed region to three islands with narrow $\Delta m_{41}^{2}$ widths and $0.00048 \lesssim \sin ^{2} 2 \vartheta_{e \mu} \lesssim$ 0.0020 at $3 \sigma$. The best-fit island is at $\Delta m_{41}^{2} \approx 1.7 \mathrm{eV}^{2}$. There is an island allowed at $2 \sigma$ at $\Delta m_{41}^{2} \approx 1.3 \mathrm{eV}^{2}$, and an island allowed at $3 \sigma$ at $\Delta m_{41}^{2} \approx 2.4 \mathrm{eV}^{2}$. However, as illustrated in figure 14, the ongoing and planned experiments have the possibility to cover all the 
allowed regions of the mixing parameters and we expect that they will reach in a few years a definitive conclusion on the existence of the short-baseline oscillations indicated by the LSND experiment and by the Gallium and reactor neutrino anomalies.

An interesting feature of the $3+1$ analysis of the MINOS and IceCube data is that there is a dependence on $\left|U_{\tau 4}\right|^{2}[138-145]$. We obtained the stringent bounds on the value of $\left|U_{\tau 4}\right|^{2}$ given in table 6, which are comparable to those obtained in ref. [39].

The determination of active-sterile neutrino mixing presented in this paper is of interest also for the phenomenology of long-baseline experiments [21-30], and neutrinoless double- $\beta$ decay experiments $[7,158-166]$.

We did not consider the problem of the cosmological bounds on active-sterile neutrino mixing [14], which most likely must be solved with a non-standard effect as a large lepton asymmetry [167-170] or secret interactions of the sterile neutrino mediated by a massive vector or pseudoscalar boson [171-177], which suppress the thermalization of the sterile neutrino in the early Universe.

In conclusion, this paper gives information on what are the regions of the parameter space of $3+1$ neutrino mixing which must be explored by new experiments in order to check the indications given by the LSND, Gallium and reactor anomalies. Let us emphasize the importance of an experimental confirmation of these oscillations, that would imply the existence of light sterile neutrinos. These are new particles with properties outside the realm of the Standard Model and their discovery would open a prodigious window on new low-energy physics.

\section{Acknowledgments}

We are very grateful to the NEOS Collaboration for giving us the table of $\chi^{2}$ corresponding to figure 4 of ref. [37]. The work of S.G. is supported by the Spanish grants FPA2014-58183-P, Multidark CSD2009-00064 and SEV-2014-0398 (MINECO), and PROMETEOII/2014/084 (Generalitat Valenciana). The work of C.G. and M.L. was partially supported by the research grant Theoretical Astroparticle Physics number 2012CPPYP7 under the program PRIN 2012 funded by the Italian Ministero dell'Istruzione, Università e della Ricerca (MIUR) and by the research project TAsP funded by the Instituto Nazionale di Fisica Nucleare (INFN). The work of Y.F.L. was supported in part by the National Natural Science Foundation of China under Grant Nos. 11305193 and 11135009, by the Strategic Priority Research Program of the Chinese Academy of Sciences under Grant No. XDA10010100, by the CAS Center for Excellence in Particle Physics (CCEPP).

Open Access. This article is distributed under the terms of the Creative Commons Attribution License (CC-BY 4.0), which permits any use, distribution and reproduction in any medium, provided the original author(s) and source are credited.

\section{References}

[1] LSND collaboration, C. Athanassopoulos et al., Candidate events in a search for $\bar{\nu}_{\mu} \rightarrow \bar{\nu}_{e}$ oscillations, Phys. Rev. Lett. 75 (1995) 2650 [nucl-ex/9504002] [INSPIRE]. 
[2] LSND collaboration, A. Aguilar-Arevalo et al., Evidence for neutrino oscillations from the observation of $\bar{\nu}_{e}$ appearance in a $\bar{\nu}_{\mu}$ beam, Phys. Rev. D 64 (2001) 112007 [hep-ex/0104049] [INSPIRE].

[3] J.N. Abdurashitov et al., Measurement of the response of a Ga solar neutrino experiment to neutrinos from an Ar-37 source, Phys. Rev. C 73 (2006) 045805 [nucl-ex/0512041] [INSPIRE].

[4] M. Laveder, Unbound neutrino roadmaps, Nucl. Phys. Proc. Suppl. 168 (2007) 344 [INSPIRE].

[5] C. Giunti and M. Laveder, Short-baseline active-sterile neutrino oscillations?, Mod. Phys. Lett. A 22 (2007) 2499 [hep-ph/0610352] [INSPIRE].

[6] C. Giunti and M. Laveder, Statistical significance of the Gallium anomaly, Phys. Rev. C 83 (2011) 065504 [arXiv: 1006 . 3244] [INSPIRE].

[7] C. Giunti, M. Laveder, Y.F. Li, Q.Y. Liu and H.W. Long, Update of short-baseline electron neutrino and antineutrino disappearance, Phys. Rev. D 86 (2012) 113014 [arXiv:1210.5715] [INSPIRE].

[8] G. Mention et al., The reactor antineutrino anomaly, Phys. Rev. D 83 (2011) 073006 [arXiv:1101.2755] [INSPIRE].

[9] S.M. Bilenky, C. Giunti and W. Grimus, Phenomenology of neutrino oscillations, Prog. Part. Nucl. Phys. 43 (1999) 1 [hep-ph/9812360] [INSPIRE].

[10] M.C. Gonzalez-Garcia and M. Maltoni, Phenomenology with massive neutrinos, Phys. Rept. 460 (2008) 1 [arXiv:0704.1800] [INSPIRE].

[11] J.M. Conrad et al., Sterile neutrino fits to short baseline neutrino oscillation measurements, Adv. High Energy Phys. 2013 (2013) 163897 [arXiv: 1207.4765] [INSPIRE].

[12] S. Gariazzo, C. Giunti, M. Laveder, Y.F. Li and E.M. Zavanin, Light sterile neutrinos, J. Phys. G 43 (2016) 033001 [arXiv: 1507.08204] [INSPIRE].

[13] M. Maltoni, T. Schwetz, M.A. Tortola and J.W.F. Valle, Status of global fits to neutrino oscillations, New J. Phys. 6 (2004) 122 [hep-ph/0405172] [inSPIRE].

[14] Planck collaboration, P.A.R. Ade et al., Planck 2015 results. XIII. Cosmological parameters, Astron. Astrophys. 594 (2016) A13 [arXiv:1502.01589] [INSPIRE].

[15] S.M. Bilenky and C. Giunti, Neutrinoless double-beta decay: a probe of physics beyond the standard model, Int. J. Mod. Phys. A 30 (2015) 1530001 [arXiv:1411.4791] [InSPIRE].

[16] S. Dell'Oro, S. Marcocci, M. Viel and F. Vissani, Neutrinoless double beta decay: 2015 review, Adv. High Energy Phys. 2016 (2016) 2162659 [arXiv:1601.07512] [INSPIRE].

[17] C. Giunti and E.M. Zavanin, Appearance-disappearance relation in $3+N_{s}$ short-baseline neutrino oscillations, Mod. Phys. Lett. A 31 (2015) 1650003 [arXiv:1508.03172] [INSPIRE].

[18] F. Capozzi, E. Lisi, A. Marrone, D. Montanino and A. Palazzo, Neutrino masses and mixings: status of known and unknown $3 \nu$ parameters, Nucl. Phys. B 908 (2016) 218 [arXiv: 1601.07777] [INSPIRE].

[19] I. Esteban, M.C. Gonzalez-Garcia, M. Maltoni, I. Martinez-Soler and T. Schwetz, Updated fit to three neutrino mixing: exploring the accelerator-reactor complementarity, JHEP 01 (2017) 087 [arXiv: 1611.01514] [INSPIRE].

[20] S.M. Bilenky, C. Giunti and W. Grimus, Neutrino mass spectrum from the results of neutrino oscillation experiments, Eur. Phys. J. C 1 (1998) 247 [hep-ph/9607372] [INSPIRE]. 
[21] A. de Gouvea, K.J. Kelly and A. Kobach, CP-invariance violation at short-baseline experiments in $3+1$ neutrino scenarios, Phys. Rev. D 91 (2015) 053005 [arXiv:1412.1479] [INSPIRE].

[22] N. Klop and A. Palazzo, Imprints of CP-violation induced by sterile neutrinos in T2K data, Phys. Rev. D 91 (2015) 073017 [arXiv:1412.7524] [INSPIRE].

[23] J.M. Berryman, A. de Gouvêa, K.J. Kelly and A. Kobach, Sterile neutrino at the Deep Underground Neutrino Experiment, Phys. Rev. D 92 (2015) 073012 [arXiv:1507.03986] [INSPIRE].

[24] R. Gandhi, B. Kayser, M. Masud and S. Prakash, The impact of sterile neutrinos on CP measurements at long baselines, JHEP 11 (2015) 039 [arXiv:1508.06275] [INSPIRE].

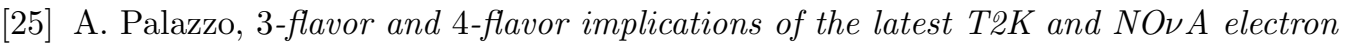
(anti-)neutrino appearance results, Phys. Lett. B 757 (2016) 142 [arXiv:1509.03148] [INSPIRE].

[26] S.K. Agarwalla, S.S. Chatterjee, A. Dasgupta and A. Palazzo, Discovery potential of T2K and NOvA in the presence of a light sterile neutrino, JHEP 02 (2016) 111 [arXiv: 1601.05995] [INSPIRE].

[27] S.K. Agarwalla, S.S. Chatterjee and A. Palazzo, Physics reach of DUNE with a light sterile neutrino, JHEP 09 (2016) 016 [arXiv:1603.03759] [INSPIRE].

[28] S. Choubey and D. Pramanik, Constraints on sterile neutrino oscillations using DUNE near detector, Phys. Lett. B 764 (2017) 135 [arXiv:1604.04731] [INSPIRE].

[29] S.K. Agarwalla, S.S. Chatterjee and A. Palazzo, Octant of $\theta_{23}$ in danger with a light sterile neutrino, Phys. Rev. Lett. 118 (2017) 031804 [arXiv: 1605.04299] [INSPIRE].

[30] F. Capozzi, C. Giunti, M. Laveder and A. Palazzo, Joint short- and long-baseline constraints on light sterile neutrinos, Phys. Rev. D 95 (2017) 033006 [arXiv:1612.07764] [INSPIRE].

[31] H.W. Long, Y.F. Li and C. Giunti, CP-violating phases in active-sterile solar neutrino oscillations, Phys. Rev. D 87 (2013) 113004 [arXiv:1304.2207] [InSPIRE].

[32] C. Giunti, M. Laveder, Y.F. Li and H.W. Long, Short-baseline electron neutrino oscillation length after Troitsk, Phys. Rev. D 87 (2013) 013004 [arXiv:1212.3805] [INSPIRE].

[33] C. Giunti, M. Laveder, Y.F. Li and H.W. Long, Pragmatic view of short-baseline neutrino oscillations, Phys. Rev. D 88 (2013) 073008 [arXiv:1308.5288] [INSPIRE].

[34] C. Giunti, Precise determination of the ${ }^{235}$ U reactor antineutrino cross section per fission, Phys. Lett. B 764 (2017) 145 [arXiv: 1608.04096] [InSPIRE].

[35] MINOS collaboration, P. Adamson et al., Search for sterile neutrinos mixing with muon neutrinos in MINOS, Phys. Rev. Lett. 117 (2016) 151803 [arXiv:1607.01176] [INSPIRE].

[36] ICECube collaboration, M.G. Aartsen et al., Searches for sterile neutrinos with the IceCube detector, Phys. Rev. Lett. 117 (2016) 071801 [arXiv: 1605. 01990] [INSPIRE].

[37] Y. Ko et al., Sterile neutrino search at the NEOS experiment, Phys. Rev. Lett. 118 (2017) 121802 [arXiv: 1610.05134] [INSPIRE].

[38] C. Giunti, Oscillations beyond three-neutrino mixing, arXiv:1609.04688 [INSPIRE].

[39] G.H. Collin, C.A. Argüelles, J.M. Conrad and M.H. Shaevitz, First constraints on the complete neutrino mixing matrix with a sterile neutrino, Phys. Rev. Lett. 117 (2016) 221801 [arXiv: 1607.00011] [INSPIRE].

[40] G.H. Collin, C.A. Argüelles, J.M. Conrad and M.H. Shaevitz, Sterile neutrino fits to short baseline data, Nucl. Phys. B 908 (2016) 354 [arXiv:1602.00671] [InSPIRE]. 
[41] Daya Bay collaboration, F.P. An et al., Improved measurement of the reactor antineutrino flux and spectrum at daya bay, Chin. Phys. C 41 (2017) 013002 [arXiv:1607.05378] [INSPIRE].

[42] N. Okada and O. Yasuda, A sterile neutrino scenario constrained by experiments and cosmology, Int. J. Mod. Phys. A 12 (1997) 3669 [hep-ph/9606411] [inSPIRE].

[43] J. Kopp, M. Maltoni and T. Schwetz, Are there sterile neutrinos at the eV scale?, Phys. Rev. Lett. 107 (2011) 091801 [arXiv:1103.4570] [INSPIRE].

[44] C. Giunti and M. Laveder, $3+1$ and $3+2$ sterile neutrino fits, Phys. Rev. D 84 (2011) 073008 [arXiv: 1107.1452] [INSPIRE].

[45] C. Giunti and M. Laveder, Status of $3+1$ neutrino mixing, Phys. Rev. D 84 (2011) 093006 [arXiv: 1109.4033] [INSPIRE].

[46] C. Giunti and M. Laveder, Implications of $3+1$ short-baseline neutrino oscillations, Phys. Lett. B 706 (2011) 200 [arXiv:1111.1069] [INSPIRE].

[47] M. Archidiacono, N. Fornengo, C. Giunti and A. Melchiorri, Testing $3+1$ and $3+2$ neutrino mass models with cosmology and short baseline experiments, Phys. Rev. D 86 (2012) 065028 [arXiv:1207.6515] [INSPIRE].

[48] M. Archidiacono, N. Fornengo, C. Giunti, S. Hannestad and A. Melchiorri, Sterile neutrinos: cosmology versus short-baseline experiments, Phys. Rev. D 87 (2013) 125034 [arXiv: 1302.6720] [INSPIRE].

[49] J. Kopp, P.A.N. Machado, M. Maltoni and T. Schwetz, Sterile neutrino oscillations: the global picture, JHEP 05 (2013) 050 [arXiv:1303.3011] [INSPIRE].

[50] MiniBoonE collaboration, A.A. Aguilar-Arevalo et al., Unexplained excess of electron-like events from a 1-GeV neutrino beam, Phys. Rev. Lett. 102 (2009) 101802 [arXiv:0812.2243] [INSPIRE].

[51] MiniBooNE collaboration, A.A. Aguilar-Arevalo et al., Improved search for $\bar{\nu}_{\mu} \rightarrow \bar{\nu}_{e}$ oscillations in the MiniBooNE experiment, Phys. Rev. Lett. 110 (2013) 161801 [arXiv: 1303.2588] [INSPIRE].

[52] MicroBooNE collaboration, S. Gollapinni, Accelerator-based short-baseline neutrino oscillation experiments, arXiv:1510.04412 [INSPIRE].

[53] Y. Declais et al., Search for neutrino oscillations at 15-meters, 40-meters and 95-meters from a nuclear power reactor at Bugey, Nucl. Phys. B 434 (1995) 503 [INSPIRE].

[54] A. Serebrov et al., Experiment neutrino-4 search for sterile neutrino with multisection detector model, PoS (INPC2016) 255 [arXiv: 1702.00941] [INSPIRE].

[55] Y. Declais et al., Study of reactor anti-neutrino interaction with proton at Bugey nuclear power plant, Phys. Lett. B 338 (1994) 383 [INSPIRE].

[56] A.A. Kuvshinnikov, L.A. Mikaelyan, S.V. Nikolaev, M.D. Skorokhvatov and A.V. Etenko, Measuring the anti-electron-neutrino $+p \rightarrow n+e^{+}$cross-section and beta decay axial constant in a new experiment at Rovno NPP reactor (in Russian), JETP Lett. 54 (1991) 253 [INSPIRE].

[57] CALTECH-SIN-TUM collaboration, G. Zacek et al., Neutrino oscillation experiments at the Gosgen nuclear power reactor, Phys. Rev. D 34 (1986) 2621 [INSPIRE].

[58] H. Kwon et al., Search for neutrino oscillations at a fission reactor, Phys. Rev. D 24 (1981) 1097 [INSPIRE].

[59] A. Hoummada et al., Neutrino oscillations I.L.L. experiment reanalysis, Appl. Radiat. Isot. 46 (1995) 449. 
[60] G.S. Vidyakin et al., Detection of anti-neutrinos in the flux from two reactors, Sov. Phys. JETP 66 (1987) 243 [INSPIRE].

[61] G.S. Vidyakin et al., Bounds on the neutrino oscillation parameters for reactor anti-neutrinos, Sov. Phys. JETP $\mathbf{7 1}$ (1990) 424 [InSPIRE].

[62] G.S. Vidyakin et al., Limitations on the characteristics of neutrino oscillations, JETP Lett. 59 (1994) 390 [INSPIRE].

[63] A.I. Afonin et al., A study of the reaction $\bar{\nu}_{e}+P \rightarrow e^{+}+N$ on a nuclear reactor, Sov. Phys. JETP 67 (1988) 213 [INSPIRE].

[64] Z.D. Greenwood et al., Results of a two position reactor neutrino oscillation experiment, Phys. Rev. D 53 (1996) 6054 [inSPIRE].

[65] P. Huber, On the determination of anti-neutrino spectra from nuclear reactors, Phys. Rev. C 84 (2011) 024617 [Erratum ibid. C 85 (2012) 029901] [arXiv:1106. 0687] [INSPIRE].

[66] Yu.V. Kozlov et al., Anti-neutrino deuteron experiment at Krasnoyarsk, Phys. Atom. Nucl. 63 (2000) 1016 [hep-ex/9912047] [INSPIRE].

[67] C. Zhang, X. Qian and P. Vogel, Reactor antineutrino anomaly with known $\theta_{13}$, Phys. Rev. D 87 (2013) 073018 [arXiv: 1303.0900] [INSPIRE].

[68] CHOOZ collaboration, M. Apollonio et al., Search for neutrino oscillations on a long baseline at the CHOOZ nuclear power station, Eur. Phys. J. C 27 (2003) 331 [hep-ex/0301017] [INSPIRE].

[69] F. Boehm et al., Final results from the Palo Verde neutrino oscillation experiment, Phys. Rev. D 64 (2001) 112001 [hep-ex/0107009] [INSPIRE].

[70] NUCIFER collaboration, G. Boireau et al., Online monitoring of the osiris reactor with the NUCIFER neutrino detector, Phys. Rev. D 93 (2016) 112006 [arXiv:1509.05610] [INSPIRE].

[71] H. Seo, Recent Results from RENO, talk presented at AAP 2016, Applied Antineutrino Physics, December 1-2, Liverpool, U.K. (2016).

[72] Yu. V. Kozlov et al., Today and future neutrino experiments at Krasnoyarsk nuclear reactor, Nucl. Phys. Proc. Suppl. 87 (2000) 514 [hep-ex/9912046] [InSPIRE].

[73] K. Schreckenbach, G. Colvin, W. Gelletly and F. Von Feilitzsch, Determination of the anti-neutrino spectrum from U-235 thermal neutron fission products up to 9.5-MeV, Phys. Lett. B 160 (1985) 325 [INSPIRE].

[74] A.A. Hahn et al., Anti-neutrino spectra from ${ }^{241} \mathrm{Pu}$ and ${ }^{239} \mathrm{Pu}$ thermal neutron fission products, Phys. Lett. B 218 (1989) 365 [INSPIRE].

[75] N. Haag, F. von Feilitzsch, L. Oberauer, W. Potzel, K. Schreckenbach and A.A. Sonzogni, Re-publication of the data from the BILL magnetic spectrometer: The cumulative $\beta$ spectra of the fission products of ${ }^{235} \mathrm{U},{ }^{239} \mathrm{Pu}$ and ${ }^{241} \mathrm{Pu}$, arXiv: 1405.3501 [INSPIRE].

[76] RENO collaboration, J.H. Choi et al., Observation of energy and baseline dependent reactor antineutrino disappearance in the RENO experiment, Phys. Rev. Lett. 116 (2016) 211801 [arXiv: 1511.05849] [INSPIRE].

[77] Double CHOOZ collaboration, Y. Abe et al., Improved measurements of the neutrino mixing angle $\theta_{13}$ with the Double CHOOZ detector, JHEP 10 (2014) 086 [Erratum ibid. 02 (2015) 074] [arXiv: 1406.7763] [INSPIRE].

[78] DaYa Bay collaboration, F.P. An et al., Measurement of the reactor antineutrino flux and spectrum at Daya Bay, Phys. Rev. Lett. 116 (2016) 061801 [arXiv:1508. 04233] [InSPIRE]. 
[79] P. Huber, Reactor antineutrino fluxes - Status and challenges, Nucl. Phys. B 908 (2016) 268 [arXiv: 1602.01499] [INSPIRE].

[80] A.C. Hayes and P. Vogel, Reactor neutrino spectra, Ann. Rev. Nucl. Part. Sci. 66 (2016) 219 [arXiv: 1605.02047] [INSPIRE].

[81] T.A. Mueller et al., Improved predictions of reactor antineutrino spectra, Phys. Rev. C 83 (2011) 054615 [arXiv:1101.2663] [inSPIRE].

[82] P. Huber, NEOS data and the origin of the $5 \mathrm{MeV}$ bump in the reactor antineutrino spectrum, Phys. Rev. Lett. 118 (2017) 042502 [arXiv: 1609.03910] [InSPIRE].

[83] C. Kraus, A. Singer, K. Valerius and C. Weinheimer, Limit on sterile neutrino contribution from the Mainz Neutrino Mass Experiment, Eur. Phys. J. C 73 (2013) 2323 [arXiv:1210.4194] [INSPIRE].

[84] A.I. Belesev et al., An upper limit on additional neutrino mass eigenstate in 2 to $100 \mathrm{eV}$ region from 'Troitsk nu-mass' data, JETP Lett. 97 (2013) 67 [arXiv:1211.7193] [INSPIRE].

[85] A.I. Belesev et al., The search for an additional neutrino mass eigenstate in the 2-100 eV region from 'Troitsk nu-mass' data: a detailed analysis, J. Phys. G 41 (2014) 015001 [arXiv: 1307.5687] [INSPIRE].

[86] C. Giunti and C.W. Kim, Fundamentals of neutrino physics and astrophysics, Oxford University Press, Oxford, U.K. (2007).

[87] C. Giunti and Y.F. Li, Matter effects in active-sterile solar neutrino oscillations, Phys. Rev. D 80 (2009) 113007 [arXiv:0910.5856] [INSPIRE].

[88] A. Palazzo, Testing the very-short-baseline neutrino anomalies at the solar sector, Phys. Rev. D 83 (2011) 113013 [arXiv:1105.1705] [INSPIRE].

[89] A. Palazzo, An estimate of $\theta_{14}$ independent of the reactor antineutrino flux determinations, Phys. Rev. D 85 (2012) 077301 [arXiv:1201.4280] [INSPIRE].

[90] A. Palazzo, Phenomenology of light sterile neutrinos: a brief review, Mod. Phys. Lett. A 28 (2013) 1330004 [arXiv:1302.1102] [INSPIRE].

[91] B. Armbruster et al., KARMEN limits on electron-neutrino $\rightarrow$ tau-neutrino oscillations in two neutrino and three neutrino mixing schemes, Phys. Rev. C 57 (1998) 3414 [hep-ex/9801007] [INSPIRE].

[92] LSND collaboration, L.B. Auerbach et al., Measurements of charged current reactions of $\nu_{e}$ on 12-C, Phys. Rev. C 64 (2001) 065501 [hep-ex/0105068] [INSPIRE].

[93] J.M. Conrad and M.H. Shaevitz, Limits on electron neutrino disappearance from the KARMEN and LSND $\nu_{e}$-carbon cross section data, Phys. Rev. D 85 (2012) 013017 [arXiv:1106.5552] [INSPIRE].

[94] T2K collaboration, K. Abe et al., Search for short baseline $\nu_{e}$ disappearance with the T2K near detector, Phys. Rev. D 91 (2015) 051102 [arXiv:1410.8811] [INSPIRE].

[95] Super-Kamiokande collaboration, K. Abe et al., Solar neutrino measurements in Super-Kamiokande-IV, Phys. Rev. D 94 (2016) 052010 [arXiv:1606.07538] [INSPIRE].

[96] Borexino collaboration, G. Bellini et al., Final results of Borexino Phase-I on low energy solar neutrino spectroscopy, Phys. Rev. D 89 (2014) 112007 [arXiv:1308.0443] [InSPIRE].

[97] Particle Data Group collaboration, C. Patrignani et al., Review of particle physics, Chin. Phys. C 40 (2016) 100001 [inSPIRE].

[98] I. Alekseev et al., DANSS: Detector of the reactor AntiNeutrino based on Solid Scintillator, 2016 JINST 11 P11011 [arXiv: 1606.02896] [INSPIRE]. 
[99] PROSPECT collaboration, J. Ashenfelter et al., The PROSPECT physics program, J. Phys. G 43 (2016) 113001 [arXiv: 1512.02202] [INSPIRE].

[100] I. Michiels, SoLid: Search for Oscillation with a 6 Li Detector at the BR2 research reactor, arXiv: 1605.00215 [INSPIRE].

[101] L. Manzanillas, STEREO: Search for sterile neutrinos at the ILL, arXiv:1702.02498 [INSPIRE].

[102] Borexino collaboration, G. Bellini et al., SOX: Short distance neutrino Oscillations with BoreXino, JHEP 08 (2013) 038 [arXiv: 1304.7721] [INSPIRE].

[103] V. Barinov, V. Gavrin, D. Gorbunov and T. Ibragimova, BEST sensitivity to $O(1) \mathrm{eV}$ sterile neutrino, Phys. Rev. D 93 (2016) 073002 [arXiv:1602.03826] [INSPIRE].

[104] A.P. Serebrov et al., NEUTRINO4 experiment: preparations for search for sterile neutrino at $100 \mathrm{MW}$ reactor $S M-3$ at 6-12 meters, arXiv:1205.2955 [INSPIRE].

[105] SoLid collaboration, N. Ryder, First results of the deployment of a SoLid detector module at the SCK-CEN BR2 reactor, PoS (EPS-HEP2015) 071 [arXiv: 1510.07835] [INSPIRE].

[106] STEREO collaboration, V. Hélaine, Sterile neutrino search at the ILL nuclear reactor: the STEREO experiment, arXiv: 1604.08877 [INSPIRE].

[107] M. Abs et al., IsoDAR@KamLAND: a conceptual design report for the technical facility, arXiv: 1511.05130 [INSPIRE].

[108] E. Ciuffoli, J. Evslin and F. Zhao, Neutrino physics with accelerator driven subcritical reactors, JHEP 01 (2016) 004 [arXiv: 1509. 03494] [INSPIRE].

[109] G. Drexlin, KATRIN, talk presented at NOW 2016, September 4-11, Otranto, Lecce, Italy (2016).

[110] A.S. Riis and S. Hannestad, Detecting sterile neutrinos with KATRIN like experiments, JCAP 02 (2011) 011 [arXiv: 1008.1495] [InSPIRE].

[111] J.A. Formaggio and J. Barrett, Resolving the reactor neutrino anomaly with the KATRIN neutrino experiment, Phys. Lett. B 706 (2011) 68 [arXiv:1105.1326] [INSPIRE].

[112] A. Sejersen Riis, S. Hannestad and C. Weinheimer, Analysis of simulated data for the KArlsruhe TRItium Neutrino experiment using Bayesian inference, Phys. Rev. C 84 (2011) 045503 [arXiv: 1105.6005] [inSPIRE].

[113] A. Esmaili and O.L.G. Peres, KATRIN sensitivity to sterile neutrino mass in the shadow of lightest neutrino mass, Phys. Rev. D 85 (2012) 117301 [arXiv:1203.2632] [INSPIRE].

[114] L. Gastaldo, C. Giunti and E.M. Zavanin, Light sterile neutrino sensitivity of $163 \mathrm{Ho}$ experiments, JHEP 06 (2016) 061 [arXiv:1605.05497] [INSPIRE].

[115] L. Borodovsky et al., Search for muon-neutrino oscillations muon-neutrino oscillations $\nu_{\mu} \rightarrow \nu_{e}\left(\bar{\nu}_{\mu} \rightarrow \bar{\mu}_{e}\right)$ in a wide band neutrino beam, Phys. Rev. Lett. 68 (1992) 274 [INSPIRE].

[116] KARMEN collaboration, B. Armbruster et al., Upper limits for neutrino oscillations $\bar{\nu}_{\mu} \rightarrow \bar{\nu}_{e}$ from muon decay at rest, Phys. Rev. D 65 (2002) 112001 [hep-ex/0203021] [INSPIRE].

[117] NOMAD collaboration, P. Astier et al., Search for $\nu_{\mu} \rightarrow \nu_{e}$ oscillations in the NOMAD experiment, Phys. Lett. B 570 (2003) 19 [hep-ex/0306037] [INSPIRE].

[118] ICARUS collaboration, M. Antonello et al., Search for anomalies in the $\nu_{e}$ appearance from a $\nu_{\mu}$ beam, Eur. Phys. J. C 73 (2013) 2599 [arXiv:1307.4699] [INSPIRE].

[119] OPERA collaboration, N. Agafonova et al., Search for $\nu_{\mu} \rightarrow \nu_{e}$ oscillations with the OPERA experiment in the CNGS beam, JHEP 07 (2013) 004 [arXiv:1303.3953] [INSPIRE]. 
[120] F. Dydak et al., A search for $\nu_{\mu}$ oscillations in the $\Delta m^{2}$ range $0.3 \mathrm{eV}^{2}$ to $90 \mathrm{eV}^{2}$, Phys. Lett. B 134 (1984) 281 [INSPIRE].

[121] M. Maltoni and T. Schwetz, Sterile neutrino oscillations after first MiniBooNE results, Phys. Rev. D 76 (2007) 093005 [arXiv: 0705. 0107] [InSPIRE].

[122] SciBoone, MiniBoone collaboration, K.B.M. Mahn et al., Dual baseline search for muon neutrino disappearance at $0.5 \mathrm{eV}^{2}<\Delta m^{2}<40 \mathrm{eV}^{2}$, Phys. Rev. D 85 (2012) 032007 [arXiv:1106.5685] [INSPIRE].

[123] SciBoone, MiniBoone collaboration, G. Cheng et al., Dual baseline search for muon antineutrino disappearance at $0.1 \mathrm{eV}^{2}<\Delta m^{2}<10 \mathrm{eV}^{2}$, Phys. Rev. D 86 (2012) 052009 [arXiv:1208.0322] [INSPIRE].

[124] M.C. Gonzalez-Garcia, F. Halzen and M. Maltoni, Physics reach of high-energy and high-statistics IceCube atmospheric neutrino data, Phys. Rev. D 71 (2005) 093010 [hep-ph/0502223] [INSPIRE].

[125] ICECuBE collaboration, M.G. Aartsen et al., Evidence for astrophysical muon neutrinos from the northern sky with IceCube, Phys. Rev. Lett. 115 (2015) 081102 [arXiv: 1507.04005] [INSPIRE].

[126] ICECube collaboration, M.G. Aartsen et al., Atmospheric and astrophysical neutrinos above 1 TeV interacting in IceCube, Phys. Rev. D 91 (2015) 022001 [arXiv:1410.1749] [InSPIRE].

[127] C.A. Argüelles Delgado, J. Salvado and C.N. Weaver, A Simple Quantum Integro-Differential Solver (SQuIDS), Comput. Phys. Commun. 196 (2015) 569 [arXiv:1412.3832] [INSPIRE].

[128] M. Honda, T. Kajita, K. Kasahara and S. Midorikawa, Calculation of the flux of atmospheric neutrinos, Phys. Rev. D 52 (1995) 4985 [hep-ph/9503439] [INSPIRE].

[129] M. Honda, T. Kajita, K. Kasahara and S. Midorikawa, A new calculation of the atmospheric neutrino flux in a 3-dimensional scheme, Phys. Rev. D 70 (2004) 043008 [astro-ph/0404457] [INSPIRE].

[130] T. Sanuki, M. Honda, T. Kajita, K. Kasahara and S. Midorikawa, Study of cosmic ray interaction model based on atmospheric muons for the neutrino flux calculation, Phys. Rev. D 75 (2007) 043005 [astro-ph/0611201] [INSPIRE].

[131] M. Honda, T. Kajita, K. Kasahara, S. Midorikawa and T. Sanuki, Calculation of atmospheric neutrino flux using the interaction model calibrated with atmospheric muon data, Phys. Rev. D 75 (2007) 043006 [astro-ph/0611418] [INSPIRE].

[132] T.K. Gaisser, T. Stanev and S. Tilav, Cosmic ray energy spectrum from measurements of air showers, Front. Phys. (Beijing) 8 (2013) 748 [arXiv:1303.3565] [INSPIRE].

[133] A.M. Dziewonski and D.L. Anderson, Preliminary reference Earth model, Phys. Earth Planet. Interiors 25 (1981) 297.

[134] C.A. Argüelles, F. Halzen, L. Wille, M. Kroll and M.H. Reno, High-energy behavior of photon, neutrino and proton cross sections, Phys. Rev. D 92 (2015) 074040 [arXiv: 1504.06639] [INSPIRE].

[135] C.A. Arguelles, New physics with atmospheric neutrinos, Ph.D. thesis, University of Wisconsin, U.S.A. (2015).

[136] B.J.P. Jones, Sterile neutrinos in cold climates, Ph.D. thesis, Massachusetts Institute of Technology (MIT), Cambridge, U.S.A. (2015).

[137] J.A. Nelder and R. Mead, A simplex method for function minimization, Comput. J. 7 (1965) 308. 
[138] H. Nunokawa, O.L.G. Peres and R. Zukanovich Funchal, Probing the LSND mass scale and four neutrino scenarios with a neutrino telescope, Phys. Lett. B 562 (2003) 279 [hep-ph/0302039] [INSPIRE].

[139] S. Choubey, Signature of sterile species in atmospheric neutrino data at neutrino telescopes, JHEP 12 (2007) 014 [arXiv:0709.1937] [INSPIRE].

[140] S. Razzaque and A. Yu. Smirnov, Searching for sterile neutrinos in ice, JHEP 07 (2011) 084 [arXiv: 1104.1390] [INSPIRE].

[141] S. Razzaque and A.Yu. Smirnov, Searches for sterile neutrinos with IceCube DeepCore, Phys. Rev. D 85 (2012) 093010 [arXiv:1203.5406] [INSPIRE].

[142] A. Esmaili, F. Halzen and O.L.G. Peres, Constraining sterile neutrinos with AMANDA and IceCube atmospheric neutrino data, JCAP 11 (2012) 041 [arXiv:1206.6903] [INSPIRE].

[143] A. Esmaili and A.Yu. Smirnov, Restricting the LSND and MiniBooNE sterile neutrinos with the IceCube atmospheric neutrino data, JHEP 12 (2013) 014 [arXiv:1307.6824] [INSPIRE].

[144] A. Esmaili, F. Halzen and O.L.G. Peres, Exploring $\nu_{\tau}-n u_{s}$ mixing with cascade events in DeepCore, JCAP 07 (2013) 048 [arXiv: 1303.3294] [InSPIRE].

[145] M. Lindner, W. Rodejohann and X.-J. Xu, Sterile neutrinos in the light of IceCube, JHEP 01 (2016) 124 [arXiv: 1510.00666] [InSPIRE].

[146] Super-Kamiokande collaboration, K. Abe et al., Limits on sterile neutrino mixing using atmospheric neutrinos in Super-Kamiokande, Phys. Rev. D 91 (2015) 052019 [arXiv:1410.2008] [INSPIRE].

[147] ICECube collaboration, M.G. Aartsen et al., Search for sterile neutrino mixing using three years of IceCube DeepCore data, arXiv:1702.05160 [INSPIRE].

[148] MINOS collaboration, P. Adamson et al., Search for sterile neutrino mixing in the MINOS long baseline experiment, Phys. Rev. D 81 (2010) 052004 [arXiv:1001.0336] [INSPIRE].

[149] MINOS collaboration, P. Adamson et al., Active to sterile neutrino mixing limits from neutral-current interactions in MINOS, Phys. Rev. Lett. 107 (2011) 011802 [arXiv: 1104.3922] [INSPIRE].

[150] OPERA collaboration, N. Agafonova et al., Limits on muon-neutrino to tau-neutrino oscillations induced by a sterile neutrino state obtained by OPERA at the CNGS beam, JHEP 06 (2015) 069 [arXiv:1503.01876] [INSPIRE].

[151] M. Martini, M. Ericson and G. Chanfray, Neutrino energy reconstruction problems and neutrino oscillations, Phys. Rev. D 85 (2012) 093012 [arXiv:1202.4745] [INSPIRE].

[152] M. Martini, M. Ericson and G. Chanfray, Energy reconstruction effects in neutrino oscillation experiments and implications for the analysis, Phys. Rev. D 87 (2013) 013009 [arXiv:1211.1523] [INSPIRE].

[153] M. Ericson, M.V. Garzelli, C. Giunti and M. Martini, Assessing the role of nuclear effects in the interpretation of the MiniBooNE low-energy anomaly, Phys. Rev. D 93 (2016) 073008 [arXiv: 1602.01390] [INSPIRE].

[154] LAr1-ND, ICARUS-WA104, MicroBooNE collaboration, M. Antonello et al., A proposal for a three detector short-baseline neutrino oscillation program in the Fermilab booster neutrino beam, arXiv:1503.01520 [INSPIRE].

[155] NUPRISM collaboration, S. Bhadra et al., Letter of intent to construct a nuPRISM detector in the J-PARC neutrino beamline, arXiv:1412.3086 [INSPIRE].

[156] JSNS2 collaboration, M. Harada et al., Proposal: a search for sterile neutrino at J-PARC materials and life science experimental facility, arXiv:1310.1437 [INSPIRE]. 
[157] S.N. Axani, G. Collin, J.M. Conrad, M.H. Shaevitz, J. Spitz and T. Wongjirad, KPipe: a decisive test for muon neutrino disappearance, arXiv:1510.06994 [INSPIRE].

[158] J. Barry, W. Rodejohann and H. Zhang, Light sterile neutrinos: models and phenomenology, JHEP 07 (2011) 091 [arXiv: 1105.3911] [INSPIRE].

[159] Y.F. Li and S.-s. Liu, Vanishing effective mass of the neutrinoless double beta decay including light sterile neutrinos, Phys. Lett. B 706 (2012) 406 [arXiv:1110.5795] [INSPIRE].

[160] W. Rodejohann, Neutrinoless double beta decay and neutrino physics, J. Phys. G 39 (2012) 124008 [arXiv: 1206. 2560] [INSPIRE].

[161] I. Girardi, A. Meroni and S.T. Petcov, Neutrinoless double beta decay in the presence of light sterile neutrinos, JHEP 11 (2013) 146 [arXiv: 1308.5802] [INSPIRE].

[162] S. Pascoli, M. Mitra and S. Wong, Effect of cancellation in neutrinoless double beta decay, Phys. Rev. D 90 (2014) 093005 [arXiv:1310.6218] [InSPIRE].

[163] A. Meroni and E. Peinado, The quest for neutrinoless double beta decay: pseudo-Dirac, Majorana and sterile neutrinos, Phys. Rev. D 90 (2014) 053002 [arXiv:1406.3990] [INSPIRE].

[164] A. Abada, V. De Romeri and A.M. Teixeira, Effect of steriles states on lepton magnetic moments and neutrinoless double beta decay, JHEP 09 (2014) 074 [arXiv:1406.6978] [INSPIRE].

[165] C. Giunti and E.M. Zavanin, Predictions for neutrinoless double-beta decay in the $3+1$ sterile neutrino scenario, JHEP 07 (2015) 171 [arXiv: 1505.00978] [INSPIRE].

[166] H. Päs and W. Rodejohann, Neutrinoless double beta decay, New J. Phys. 17 (2015) 115010 [arXiv: 1507.00170] [INSPIRE].

[167] S. Hannestad, I. Tamborra and T. Tram, Thermalisation of light sterile neutrinos in the early universe, JCAP 07 (2012) 025 [arXiv: 1204.5861] [INSPIRE].

[168] A. Mirizzi, N. Saviano, G. Miele and P.D. Serpico, Light sterile neutrino production in the early universe with dynamical neutrino asymmetries, Phys. Rev. D 86 (2012) 053009 [arXiv:1206.1046] [INSPIRE].

[169] N. Saviano, A. Mirizzi, O. Pisanti, P.D. Serpico, G. Mangano and G. Miele, Multi-momentum and multi-flavour active-sterile neutrino oscillations in the early universe: role of neutrino asymmetries and effects on nucleosynthesis, Phys. Rev. D 87 (2013) 073006 [arXiv: 1302.1200] [INSPIRE].

[170] S. Hannestad, R.S. Hansen and T. Tram, Can active-sterile neutrino oscillations lead to chaotic behavior of the cosmological lepton asymmetry?, JCAP 04 (2013) 032 [arXiv: 1302.7279] [INSPIRE].

[171] S. Hannestad, R.S. Hansen and T. Tram, How self-interactions can reconcile sterile neutrinos with cosmology, Phys. Rev. Lett. 112 (2014) 031802 [arXiv:1310.5926] [INSPIRE].

[172] B. Dasgupta and J. Kopp, Cosmologically safe eV-scale sterile neutrinos and improved dark matter structure, Phys. Rev. Lett. 112 (2014) 031803 [arXiv:1310.6337] [INSPIRE].

[173] A. Mirizzi, G. Mangano, O. Pisanti and N. Saviano, Collisional production of sterile neutrinos via secret interactions and cosmological implications, Phys. Rev. D 91 (2015) 025019 [arXiv: 1410.1385 ] [INSPIRE]. 
[174] N. Saviano, O. Pisanti, G. Mangano and A. Mirizzi, Unveiling secret interactions among sterile neutrinos with big-bang nucleosynthesis, Phys. Rev. D 90 (2014) 113009 [arXiv: 1409.1680] [INSPIRE].

[175] F. Forastieri, M. Lattanzi and P. Natoli, Constraints on secret neutrino interactions after Planck, JCAP 07 (2015) 014 [arXiv: 1504.04999] [INSPIRE].

[176] X. Chu, B. Dasgupta and J. Kopp, Sterile neutrinos with secret interactions-lasting friendship with cosmology, JCAP 10 (2015) 011 [arXiv:1505.02795] [INSPIRE].

[177] M. Archidiacono et al., Pseudoscalar-sterile neutrino interactions: reconciling the cosmos with neutrino oscillations, JCAP 08 (2016) 067 [arXiv: 1606.07673] [INSPIRE].

[178] M. Maltoni and T. Schwetz, Testing the statistical compatibility of independent data sets, Phys. Rev. D 68 (2003) 033020 [hep-ph/0304176] [INSPIRE]. 